\title{
Nonextensive Tsallis statistics in Unruh effect for Dirac neutrinos
}

\author{
Giuseppe Gaetano Luciano $^{1,2, \mathrm{a}}{ }_{\mathbb{D}}$, Massimo Blasone $\mathrm{e}^{1,2, \mathrm{~b}}$ \\ ${ }_{1}^{1}$ Dipartimento di Fisica, Università di Salerno, Via Giovanni Paolo II, 132, 84084 Fisciano, SA, Italy \\ ${ }^{2}$ INFN, Sezione di Napoli, Gruppo collegato di Salerno, Via Giovanni Paolo II, 132, 84084 Fisciano, SA, Italy
}

Received: 12 September 2021 / Accepted: 29 October 2021 / Published online: 12 November 2021

(c) The Author(s) 2021

\begin{abstract}
Flavor mixing of quantum fields was found to be responsible for the breakdown of the thermality of Unruh effect. Recently, this result was revisited in the context of nonextensive Tsallis thermostatistics, showing that the emergent vacuum condensate can still be featured as a thermallike bath, provided that the underlying statistics is assumed to obey Tsallis prescription. This was analyzed explicitly for bosons. Here we extend this study to Dirac fermions and in particular to neutrinos. Working in the relativistic approximation, we provide an effective description of the modified Unruh spectrum in terms of the $q$-generalized Tsallis statistics, the $q$-entropic index being dependent on the mixing parameters $\sin \theta$ and $\Delta m$. As opposed to bosons, we find $q>1$, which is indicative of the subadditivity regime of Tsallis entropy. An intuitive understanding of this result is discussed in relation to the nontrivial entangled structure exhibited by the quantum vacuum for mixed fields, combined with the Pauli exclusion principle.
\end{abstract}

\section{Introduction}

The Unruh effect states that an observer moving through the inertial vacuum with uniform acceleration $a$ (Rindler observer) perceives a thermal radiation at temperature [1].

$T_{\mathrm{U}}=a / 2 \pi$,

in natural units. Since its theoretical prediction, such an effect has stimulated deep investigation, including applications to quantum optics and quantum information [2-5], connections with cosmological scenarios [6-8] and the interplay with other genuinely field theoretical phenomena [9-20] (see [21] for a review). Furthermore, experimental proposals have been suggested in a variety of contexts, ranging from direct attempts in particle physics [22,23] to analog models in water

\footnotetext{
a e-mail: gluciano@sa.infn.it (corresponding author)

be-mail: blasone@sa.infn.it
}

[24,25] and condensed matter systems [26-28]. In spite of these efforts, the problem of revealing the Unruh radiation is still open.

Recently, the growing interest in the Unruh effect has led to unveil unconventional features of this phenomenon. For instance, in [29] it was argued that the Casimir-Polder forces between two relativistic uniformly accelerated atoms exhibit a transition from the short distance thermal-like behavior predicted by the Unruh effect to a long distance non-thermal behavior. Similarly, in [20] the Unruh temperature $T_{\mathrm{U}}$ was claimed to be sensitive to the degree of departure from linearity of the dispersion relation for the case of massive fields. Exotic behaviors of Unruh radiation also appear when considering the superposition of fields with different masses, as stated in [30,31]. Specifically, in [30] it was shown that the vacuum density of mixed particles detected by the Rindler observer deviates from the pure Planckian spectrum, the correction being quantified by the mass difference and the mixing angle. In turn, this feature can be ascribed to the peculiar nature of the vacuum for mixed fields (flavor vacuum), which appears as a condensate of entangled particle/antiparticle pairs due to the nontrivial structure of the mixing transformations at level of the ladder operators [32,33].

The mixing-induced departure of Unruh condensate from the Planckian profile was originally interpreted as a breakdown of the thermality of Unruh effect [30]. In a recent work [34], this result has been revisited in the context of the $q$ generalized Tsallis statistics based on the nonadditive Tsallis entropy [35-38]. As well-known, Tsallis entropy is a generalization of the standard Boltzmann-Gibbs definition parameterized by the $q$-index (in particular, $q \neq 1$ corresponds to the nonextensive Tsallis statistics, while $q \rightarrow 1$ gives back Boltzmann-Gibbs framework). The concept was introduced as a basis for extending the usual statistical mechanics and is formally identical to Havrda-Charvát structural $\alpha$-entropy considered in the information theory [39]. The rationale behind the study of the modified Unruh effect within Tsallis statistics is that the $q$-generalized entropy well describes 
systems exhibiting long-range interactions and/or spacetime entanglement, as is the case for mixed fields $[32,33,40,41]$. In this vein, in [34] it has been shown that the Unruh condensate for mixed particles can still be described as a thermal-like distribution, provided that the underlying statistics is assumed to obey Tsallis's prescription. This picture allows to relate the $q$-entropic index and the characteristic mixing parameters $\sin \theta$ and $\Delta m$ in a nontrivial way. Clearly, in the absence of mixing (i.e. for vanishing $\theta$ and/or $\Delta m$ ), the BoltzmannGibbs theory is recovered. In passing, we mention that applications of Tsallis entropy have been widely considered in literature. Among the various systems that clarify the physical conditions under which Tsallis entropy and the associated statistics apply, we quote self-gravitating stellar systems $[42,43]$, black holes [37], the cosmic background radiation $[44,45]$, low-dimensional dissipative systems [38], solar neutrinos [46], polymer chains [47] and cosmological models $[48,49]$.

The description of Unruh effect in Tsallis's theory has been developed for mixing of boson fields. In that case, it has been found that $q<1$, indicating a superadditive behavior of Tsallis entropy. In what follows, we extend this study to the case of Dirac fermions and in particular to neutrinos, which are the paradigmatic example of mixed particles. Again, we relate the $q$-index to the mixing angle and the mass difference, in such a way that the standard FermiDirac distribution based on the extensive Boltzmann-Gibbs entropy is restored for vanishing mixing. Similarly to [34], we find a running behavior of $q$ as a function of the energy scale, which is typical for QFT systems [48]. Nevertheless, as opposed to bosons, we now obtain $q>1$, that is to say, the entropy function turns out to be subadditive. Following [34], we explore our result in connection with the entangled condensate structure exhibited by the quantum vacuum for mixed fields.

The remainder of the work is organized as follows: in Sect. 2 we review the quantization of Dirac field in Rindler spacetime and the origin of the Unruh effect. Section 3 is devoted to the study of the QFT formalism of field mixing in Rindler spacetime. This prepares the ground for the analysis of Sect. 4, where we introduce the framework of Tsallis thermodynamics and investigate the connection with the phenomenon of field mixing. To avoid technicalities, we make use of some approximations, such as those of relativistic neutrinos and small deviations of $q$ from unity. Discussion of the results and outlook are summarized in Sect. 5. Two Appendices containing details of calculations conclude the work. Throughout all the manuscript, we use the 4-dimensional metric with the mostly negative signature

$\eta_{\mu \nu}=(+1,-1,-1,-1)$.

Furthermore we adopt natural units $\hbar=c=k_{\mathrm{B}}=1$.

\section{Field quantization in Rindler spacetime: the Unruh effect}

Let us consider an observer moving in Minkowski spacetime along the $x$-axis with constant proper acceleration $a>0$. From [50], it is known that his trajectory is given by

$t=a^{-1} \sinh (a \tau), \quad x=a^{-1} \cosh (a \tau)$,

$y=y(0), \quad z=z(0)$,

parameterized by $\tau$. It is easy to see that this is an hyperbola in the $(t, x)$-plane of asymptotes $x= \pm t$, which act as event horizons for the accelerated observer. By varying $a$, we obtain different hyperbolas with the same geometric (and, thus, physical) properties.

Starting from the Minkowski metric $d s^{2}=d t^{2}-d x^{2}-$ $d y^{2}-d z^{2}$, we now introduce the following set of coordinates

$t=\rho \sinh \eta, \quad x=\rho \cosh \eta$,

$\rho=\sqrt{x^{2}-t^{2}}, \quad \eta=\operatorname{arctgh}(t / x)$,

with the other coordinates left unchanged. The metric then becomes

$d s^{2}=\rho^{2} d \eta^{2}-d \rho^{2}-d y^{2}-d z^{2}$,

which describes a stationary spacetime of timelike Killing vector $\partial / \partial \eta$.

By comparing Eqs. (3) and (4), the wordlines $\rho=$ const, $y=$ const, $z=$ const correspond to the trajectories of uniformly accelerated observers with $a=\rho^{-1}$ and proper time $\tau=a \eta$. The collection of these worldlines forms the socalled Rindler spacetime (see the right $R$-wedge in Fig. 1). Notice that the hypersurfaces of $\eta=$ const describe events which are simultaneous from the point of view of the Rindler (uniformly accelerated) observer.

In spite of the minimalistic setting, the geometric structure of Rindler manifold differs significantly from that of Minkowski spacetime. Indeed, an observer moving along a fixed hyperbola in $R$ is causally disconnected from the $L$ sector, which means that he can neither send nor receive any signal from a source in that region. Of course, this does not apply to inertial (Minkowski) observers, which are indeed connected to the entire spacetime.

Strictly speaking, the set of coordinates (4)-(5) covers only the right sector of Minkowski spacetime. To describe the left $L$-wedge, one has to require $\rho=-\sqrt{x^{2}-t^{2}}<0$, with $\eta$ running in the opposite direction. On the other hand, the future $F$ and past $P$ regions are covered by the charts

$t=\rho \cosh \eta, \quad x=\rho \sinh \eta$,

$\rho= \pm \sqrt{t^{2}-x^{2}}, \quad \eta=\operatorname{arctgh}(x / t)$,

where the \pm signs refer to $F$ and $P$, respectively. We emphasize that these charts are obtained by inverting the rôles of 


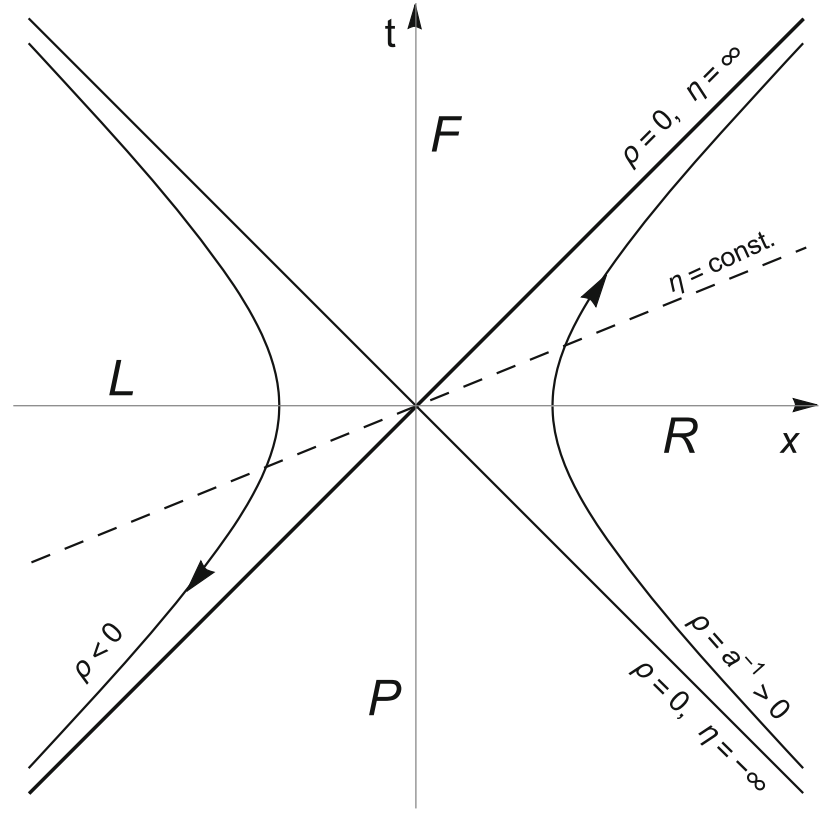

Fig. 1 Causal structure of Rindler metric in $1+1$ dimensions. We are assuming that the acceleration is along the $x$-axis. The right branch of hyperbola represents the worldline of the Rindler observer. The arrow indicates the direction of motion

time and space coordinates in Eq. (4). For our next purposes, it is enough to consider the right and left wedges only.

\subsection{Minkowski spacetime}

Let us now turn to the problem of quantizing the Dirac field in Rindler spacetime. Toward this end, it is useful to review the canonical planewave expansion in Minkowski space. This is given by

$\Psi(t, \mathbf{x})=\sum_{r=1,2} \int d^{3} k\left[a_{\mathbf{k}}^{r} \psi_{\mathbf{k}}^{r+}(t, \mathbf{x})+b_{\mathbf{k}}^{r \dagger} \psi_{\mathbf{k}}^{r-}(t, \mathbf{x})\right]$,

where $\mathbf{x} \equiv(x, y, z)$ denotes the set of spatial Minkowski coordinates. Here, $\psi_{\mathbf{k}}^{r+}=N u_{\mathbf{k}}^{r} e^{-i \omega_{\mathbf{k}} t+i \mathbf{k} \cdot \mathbf{x}}$ and $\psi_{\mathbf{k}}^{r-}=$ $N v_{\mathbf{k}}^{r} e^{i \omega_{\mathbf{k}} t-i \mathbf{k} \cdot \mathbf{x}}$ are the positive and negative frequency modes with three-momentum $\mathbf{k} \equiv\left(k_{x}, k_{y}, k_{z}\right)$ and frequency $\omega_{\mathbf{k}}=$ $\sqrt{m^{2}+|\mathbf{k}|^{2}}$, respectively. The normalization coefficient is fixed to $N=(2 \pi)^{-3 / 2}$. The following explicit form of the spinors $u_{\mathbf{k}}^{r}$ and $v_{\mathbf{k}}^{r}$ is adopted

$u_{\mathbf{k}}^{r}=\frac{\not p+m}{\sqrt{2 \omega_{\mathbf{k}}\left(\omega_{\mathbf{k}}+m\right)}} u^{r}(0), \quad v_{\mathbf{k}}^{r}=\frac{-\not p+m}{\sqrt{2 \omega_{\mathbf{k}}\left(\omega_{\mathbf{k}}+m\right)}} v^{r}(0)$,

where we have used the usual notation $\not p=p^{\mu} \gamma_{\mu}$, with $\gamma^{\mu}$ being the gamma matrices in the Dirac representation and $u^{r}(0), v^{r}(0)$ the unit spinors in the particle rest frame of $\mathbf{k}=(m, 0)$ (see [51]). They are normalized according to

$u_{\mathbf{k}}^{r \dagger} u_{\mathbf{k}}^{s}=\delta_{r s}$, and similarly for $v_{\mathbf{k}}^{r}$.

In the second quantization formalism the operators $a_{\mathbf{k}}^{r}$ and $b_{\mathbf{k}}^{r}$ act as annihilators of the ordinary Minkowski vacuum $|0\rangle_{\mathrm{M}}$, i.e.

$a_{\mathbf{k}}^{r}|0\rangle_{\mathrm{M}}=b_{\mathbf{k}}^{r}|0\rangle_{\mathrm{M}}=0, \quad \forall \mathbf{k}, r$,

while $a_{\mathbf{k}}^{r \dagger}$ and $b_{\mathbf{k}}^{r \dagger}$ create a particle/antiparticle with quantum numbers $\mathbf{k}$ and $r$. Such operators are assumed to satisfy the canonical anticommutation relations $\left\{a_{\mathbf{k}}^{r}, a_{\mathbf{k}^{\prime}}^{s^{\dagger}}\right\}=$ $\left\{b_{\mathbf{k}}^{r}, b_{\mathbf{k}^{\prime}}^{s^{\dagger}}\right\}=\delta_{r s} \delta\left(\mathbf{k}-\mathbf{k}^{\prime}\right)$, with all other anticommutators vanishing. In turn, this implies the following relation for the equal-time anticommutator between the field and its hermitian conjugate

$$
\left\{\Psi_{a}(t, \mathbf{x}), \Psi_{\beta}^{\dagger}\left(t, \mathbf{x}^{\prime}\right)\right\}=\delta_{\alpha \beta} \delta^{3}\left(\mathbf{x}-\mathbf{x}^{\prime}\right)
$$

where $\alpha$ and $\beta$ are the spinorial indices.

\subsection{Rindler spacetime}

We now properly aim at performing the second quantization of the spinor field in Rindler spacetime. To fix the notation, we closely follow [52] and consider the solutions of the Dirac equation in Rindler coordinates in the $R$-wedge. We emphasize that these modes can be extended to the remaining sectors $F, L$ and $P$ via analytic continuation across the event horizons, which represent branch points for these functions.

By using Eq. (4), the Dirac equation in $R$ takes the form

$$
\begin{gathered}
\left(i \partial_{\eta}+i \rho \gamma^{0} \gamma^{1} \partial_{\rho}+i \rho \gamma^{0} \gamma^{2} \partial_{y}+i \rho \gamma^{0} \gamma^{3} \partial_{z}\right. \\
\left.+\frac{i}{2} \gamma^{0} \gamma^{1}-m \rho \gamma^{0}\right) \psi(\eta, \rho, \vec{x})=0 .
\end{gathered}
$$

This admits as solutions

$$
\begin{aligned}
\Psi_{1, \kappa}(\eta, \rho, \vec{x})= & N_{\kappa}\left(X_{1, \vec{k}} K_{i \Omega-\frac{1}{2}}\left(\mu_{\vec{k}} \rho\right)\right. \\
& \left.+Y_{1, \vec{k}} K_{i \Omega+\frac{1}{2}}\left(\mu_{\vec{k}} \rho\right)\right) e^{-i \Omega \eta} e^{i \vec{k} \cdot \vec{x}}
\end{aligned}
$$

with

$X_{1, \vec{k}}=\left(\begin{array}{c}k_{z} \\ i\left(k_{y}+i m\right) \\ i\left(k_{y}+i m\right) \\ k_{z}\end{array}\right), \quad Y_{1, \vec{k}}=\left(\begin{array}{c}0 \\ i \mu_{\vec{k}} \\ -i \mu_{\vec{k}} \\ 0\end{array}\right)$

and

$$
\begin{aligned}
\Psi_{2, \kappa}(\eta, \rho, \vec{x})= & N_{\kappa}\left(X_{2, \vec{k}} K_{i \Omega-\frac{1}{2}}\left(\mu_{\vec{k}} \rho\right)\right. \\
& \left.+Y_{2, \vec{k}} K_{i \Omega+\frac{1}{2}}\left(\mu_{\vec{k}} \rho\right)\right) e^{-i \Omega \eta} e^{i \vec{k} \cdot \vec{x}},
\end{aligned}
$$


with

$X_{2, \vec{k}}=\left(\begin{array}{c}0 \\ i \mu_{\vec{k}} \\ i \mu_{\vec{k}} \\ 0\end{array}\right), \quad Y_{2, \vec{k}}=\left(\begin{array}{c}k_{z} \\ i\left(k_{y}-i m\right) \\ -i\left(k_{y}-i m\right) \\ -k_{z}\end{array}\right)$.

In the above equations we have used the shorthand notation $\vec{x} \equiv(y, z)$ and $\vec{k} \equiv\left(k_{y}, k_{z}\right)$ for the transverse position and momentum coordinates, respectively. The Hamiltonian eigenvalue has been denoted by $\Omega>0$, while $\kappa \equiv\{\Omega, \vec{k}\}$. The $\rho$-dependence of the Rindler modes is contained in the Bessel function $K_{i \Omega \pm \frac{1}{2}}$, where $\mu_{\vec{k}}=$ $\sqrt{m^{2}+|\vec{k}|^{2}}$ is the reduced frequency. The coefficient $N_{\kappa}=$ $\left(4 \pi^{2} \sqrt{\mu_{\vec{k}}}\right)^{-1} \sqrt{\cosh (\pi \Omega)}$ provides the normalization respect to the inner product in Rindler coordinates [52].

For later purposes, it is also useful to consider the solutions (15) and (17) expressed in Minkowski coordinates. By taking into account the spinor transformations under coordinate changes, these solutions take the form [52]

$$
\begin{aligned}
\Psi_{j, \kappa}(t, \mathbf{x})= & N_{\kappa}\left(X_{j, \vec{k}} K_{i \Omega-\frac{1}{2}}\left(\mu_{\vec{k}} \rho\right) e^{-\left(i \Omega-\frac{1}{2}\right) \eta}\right. \\
& \left.+Y_{j, \vec{k}} K_{i \Omega+\frac{1}{2}}\left(\mu_{\vec{k}} \rho\right) e^{-\left(i \Omega+\frac{1}{2}\right) \eta}\right) e^{i \vec{k} \cdot \vec{x}}, \\
& j=1,2 .
\end{aligned}
$$

We stress that these functions are still defined in the $R$-wedge only.

Exploiting the above tools, we now expand the Dirac field in $R$ as

$$
\begin{aligned}
\Psi_{R}(\eta, \rho, \vec{x})= & \sum_{j=1,2} \int d^{3} \kappa\left[a_{j, \kappa} \psi_{j, \kappa}(\eta, \rho, \vec{x})\right. \\
& \left.+b_{j, \kappa}^{\dagger} \psi_{j, \tilde{\kappa}}(\eta, \rho, \vec{x})\right],
\end{aligned}
$$

where $\tilde{\kappa} \equiv(-\Omega, \vec{k})$. As for the Minkowski quantization, the operators $a_{j, \kappa}$ and $b_{j, \kappa}$ are assumed to be canonical, i.e.

$$
\left\{a_{j, \kappa}, a_{i, \kappa^{\prime}}^{\dagger}\right\}=\left\{b_{j, \kappa}, b_{i \kappa^{\prime}}^{\dagger}\right\}=\delta_{j i} \delta^{3}\left(\kappa-\kappa^{\prime}\right),
$$

with all other anticommutators being trivial. They now annihilate the Rindler vacuum state

$a_{j, \kappa}|0\rangle_{\mathrm{R}}=b_{j, \kappa}|0\rangle_{\mathrm{R}}=0, \quad \forall \kappa, j$,

while $a_{j, \kappa}^{\dagger}$ and $b_{j, \kappa}^{\dagger}$ create a particle/antiparticle for the Rindler observer in $R$.

To make it easier the comparison between the field expansions (9) and (20), let us introduce an alternative field quantization in Minkowski spacetime, namely a quantization in terms of the Lorentz momentum eigenfunctions. The physical reason behind this formalism is that the Rindler Hamiltonian is exactly the Lorentz boost generator written in Minkowski coordinates. This means that the time evolution of a Rindler observer can be properly described as an infinite succession of infinitesimal boost transformations (in our case along the $x$-axis).

We consider the integral representations of the Bessel function $K_{v}(\rho)$ given by

$K_{\nu}(\rho)=\frac{1}{2} e^{-\frac{i \pi \nu}{2}} \int_{-\infty}^{\infty} d \theta e^{i \rho \sinh \theta} e^{\nu \theta}$,
$K_{\nu}(\rho)=\frac{1}{2} e^{\frac{i \pi \nu}{2}} \int_{-\infty}^{\infty} d \theta e^{i \rho \sinh \theta} e^{-\nu \theta}$.

From the last representation, by using the coordinate transformation (4) one can show that [52]

$K_{v}\left(\mu_{\vec{k}} \rho(t, x)\right) e^{-v \eta}=\frac{1}{2} e^{\frac{i \pi v}{2}} \int_{-\infty}^{\infty} d \theta P_{\theta}^{-}(t, x) e^{-v \theta}$,

where $P_{\theta}^{-}(t, x)$ is the two-dimensional positive-frequency planewave with $\omega=\mu_{\vec{k}} \cosh \theta$ and $k_{x}=\mu_{\vec{k}} \sinh \theta$. On the other hand, Eq. (23) leads to

$K_{\nu}\left(\mu_{\vec{k}} \rho(t, x)\right) e^{-v \eta}=\frac{1}{2} e^{-\frac{i \pi v}{2}} \int_{-\infty}^{\infty} d \theta P_{\theta}^{+}(t, x) e^{\nu \theta}$,

where $P_{\theta}^{+}(t, x)$ is the two-dimensional negative-frequency planewave. Remarkably, Eqs. (25) and (26) show that the function $K_{v}\left(\mu_{\vec{k}} \rho(t, x)\right) e^{-v \eta}$ can be expressed equivalently as superposition of either positive- or negative-frequency planewaves.

If we now plug the above representations of the Bessel function into Eq. (19), we obtain

$$
\begin{aligned}
& \psi_{j, k}^{\mp}(t, \mathbf{x})=\frac{1}{2} N_{\kappa}^{\mp}\left[X_{j, \vec{k}} e^{ \pm \frac{i \pi}{2}\left(i \Omega-\frac{1}{2}\right)} \int_{-\infty}^{\infty} d \theta P_{\theta}^{\mp}(t, x) e^{\mp\left(i \Omega-\frac{1}{2}\right) \theta}\right. \\
& \left.+Y_{j, \vec{k}} e^{ \pm \frac{i \pi}{2}\left(i \Omega+\frac{1}{2}\right)} \int_{-\infty}^{\infty} d \theta P_{\theta}^{\mp}(t, x) e^{\mp\left(i \Omega-\frac{1}{2}\right) \theta}\right] e^{i \vec{k} \cdot \vec{x}} .
\end{aligned}
$$

As opposed to Eq. (19), these functions are globally defined (and analytical) on the entire Minkowski manifold, except for the origin. Furthermore, consistently with our previous considerations, they are eigenfunctions of the boost generator, with eigenvalue $\Omega$. The coefficient $N_{\kappa}^{\mp}=\left(2 \pi \sqrt{\mu_{\vec{k}}}\right)^{-1} e^{ \pm \pi \Omega / 2}$ is now fixed by requiring the orthonormality with respect to the ordinary inner product in Minkowski spacetime. We also notice that the existence of two different integral representations of the Bessel function is reflected into the existence of two different global representations of the Rindler modes in Minkowski coordinates. ${ }^{1}$ In turn, this is related to the possibility of extending such modes across the event horizons along two different paths (see [52] for more details).

At this stage, we can exploit the fact that the functions $\psi_{j, \kappa}^{+}$ $\left(\psi_{j, \kappa}^{-}\right)$are linear combinations of only positive- (negative-)

\footnotetext{
1 The modes (27) represent the spinorial counterpart of the Gerlach's Minkowski Bessel functions for the scalar field [30,53].
} 
frequency planewaves to introduce a field expansion equivalent to Eq. (9) as regards the associated Fock space. This expansion reads

$$
\Psi(t, \mathbf{x})=\sum_{j=1,2} \int d^{3} \kappa\left[c_{j, \kappa} \psi_{j, \kappa}^{-}(t, \mathbf{x})+d_{j, \kappa}^{\dagger} \psi_{j, \kappa}^{+}(t, \mathbf{x})\right]
$$

Since a field excitation in this quantization is still a positivefrequency mode with respect to the time $t$, it is easy to understand that the vacuum for $c_{k, \kappa}$ and $d_{k, \kappa}$ is the same as that for $a_{\mathbf{k}}^{r}$ and $b_{\mathbf{k}}^{r}$ defined in Eq. (12). Mathematically, this can be seen by the following relations

$$
\begin{aligned}
& c_{j, \kappa}=\left(\psi_{j, \kappa}^{-}, \Psi\right)=\sum_{r=1,2} \int d k_{x} F_{j, r}\left(k_{x}, \Omega\right) a_{\mathbf{k}}^{r} \\
& F_{j, r}\left(k_{x}, \Omega\right)=\frac{4 \pi^{3}}{\omega_{\mathbf{k}}} N_{\kappa}^{-} N\left[\left(\frac{\omega_{\mathbf{k}}+k_{x}}{\omega_{\mathbf{k}}-k_{x}}\right)^{i \frac{\Omega}{2}+\frac{1}{4}} e^{i \frac{\pi}{2}\left(i \Omega+\frac{1}{2}\right)}\right. \\
& \left.X_{j, \vec{k}}^{\dagger}+\left(\frac{\omega_{\mathbf{k}}+k_{x}}{\omega_{\mathbf{k}}-k_{x}}\right)^{i \frac{\Omega}{2}-\frac{1}{4}} e^{i \frac{\pi}{2}\left(i \Omega-\frac{1}{2}\right)} Y_{j, \vec{k}}^{\dagger}\right] u_{\mathbf{k}}^{r}
\end{aligned}
$$

which show that the $c_{j, \kappa}$-annihilator is a linear superposition of the $a_{\mathbf{k}}^{r}$ 's only. Similarly, for $d_{j, \kappa}^{\dagger} \psi_{j, \kappa}^{+}$we have

$$
\begin{aligned}
& d_{j, \kappa}^{\dagger}=\left(\psi_{j, \kappa}^{+}, \Psi\right)=\sum_{r=1,2} \int d k_{x} G_{j, r}\left(k_{x}, \Omega\right) b_{\mathbf{k}}^{r \dagger} \\
& G_{j, r}\left(k_{x}, \Omega\right)=\frac{4 \pi^{3}}{\omega_{\mathbf{k}}} N_{\kappa}^{+} N\left[\left(\frac{\omega_{\mathbf{k}}+k_{x}}{\omega_{\mathbf{k}}-k_{x}}\right)^{i \frac{\Omega}{2}+\frac{1}{4}} e^{i \frac{\pi}{2}\left(i \Omega+\frac{1}{2}\right)}\right. \\
& \left.X_{j, \vec{k}}^{\dagger}+\left(\frac{\omega_{\mathbf{k}}+k_{x}}{\omega_{\mathbf{k}}-k_{x}}\right)^{i \frac{\Omega}{2}-\frac{1}{4}} e^{i \frac{\pi}{2}\left(i \Omega-\frac{1}{2}\right)} Y_{j, \vec{k}}^{\dagger}\right] v_{\mathbf{k}}^{r}
\end{aligned}
$$

which show that the $d_{j, \kappa}$-annihilator is a linear superposition of the $b_{\mathbf{k}}^{r}$ 's only. To simplify the notation, in the above formulas we have omitted the dependence of $F_{j, r}$ and $G_{j, r}$ by the transverse momentum $\vec{k}$, since it does not play any significant rôle in our next calculations. Furthermore, the field expansion (9) has been used as right entry for the computation of the Minkowski inner products (29) and (31).

By using Eq. (12), it goes without saying that

$c_{j, \kappa}|0\rangle_{\mathrm{M}}=d_{j, \kappa}|0\rangle_{\mathrm{M}}=0, \quad \forall \kappa, j$

The proof of the equivalence between the two quantum constructions is completed by noticing that the transformations (29) and (31) are canonical, which implies that the new ladder operators $c_{j, \kappa}$ and $d_{j, \kappa}$ still obey the canonical anticommutation relations.

\subsection{Unruh effect}

We can now derive the Unruh effect for the Dirac field as originally proposed in [1]. Such computation requires the formulation of a quantum construction which is valid for the Minkowski spacetime and gives back the quantization (20) when restricted to the $R$-wedge. For this task, we make use of the global modes $\psi_{j, \kappa}^{\mp}$ and define the two combinations

$$
\begin{aligned}
& \mathcal{R}_{j, \kappa}=\frac{1}{\sqrt{2 \cosh (\pi \Omega)}}\left(e^{\frac{\pi \Omega}{2}} \psi_{j, \kappa}^{-}+e^{-\frac{\pi \Omega}{2}} \psi_{j, \kappa}^{+}\right), \\
& \mathcal{L}_{j, \kappa}=\frac{1}{\sqrt{2 \cosh (\pi \Omega)}}\left(e^{-\frac{\pi \Omega}{2}} \psi_{j, \kappa}^{-}-e^{\frac{\pi \Omega}{2}} \psi_{j, \kappa}^{+}\right) .
\end{aligned}
$$

These functions have all the required properties. Indeed, they are analytical and orthonormal in the whole Minkowski space. Furthermore $\mathcal{R}_{j, \kappa}$ is such that it is defined everywhere but in the $L$-sector and reduces to Eqs. (15) and (17) in $R$ (depending on whether $j=1,2$ ). The opposite behavior holds for $\mathcal{L}_{j, \kappa}$.

By inverting the above relations with respect to $\psi_{j, \kappa}^{\mp}$ and replacing into Eq. (28), we finally get

$$
\begin{aligned}
\psi(t, \mathbf{x})= & \sum_{j=1,2} \int d^{3} \kappa\left[r_{j, \kappa} \mathcal{R}_{j, \kappa}(t, \mathbf{x})+r_{j, \kappa}^{\dagger} \mathcal{R}_{j, \tilde{\kappa}}(t, \mathbf{x})\right. \\
& \left.+l_{j, \kappa} \mathcal{L}_{j, \tilde{\kappa}}(t, \mathbf{x})+l_{j, \kappa}^{\dagger} \mathcal{L}_{j, \kappa}(t, \mathbf{x})\right]
\end{aligned}
$$

where the operators $r_{j, \kappa}$ and $l_{j, \kappa}$ are connected to $c_{j, \kappa}$ and $d_{j, \kappa}$ via the Bogoliubov transformations

$r_{j, \kappa}=\frac{c_{j, \kappa} e^{\frac{\pi \Omega}{2}}+d_{j, \kappa}^{\dagger} e^{-\frac{\pi \Omega}{2}}}{\sqrt{2 \cosh (\pi \Omega)}}$,

$l_{j, \kappa}^{\dagger}=\frac{c_{j, \kappa} e^{-\frac{\pi \Omega}{2}}-d_{j, \kappa}^{\dagger} e^{\frac{\pi \Omega}{2}}}{\sqrt{2 \cosh (\pi \Omega)}}$.

If we now identify $r_{j, \kappa}$ with the creation operator for the Rindler observer in $R$, we can evaluate the spectrum of particles detected by such an observer in the Minkowski vacuum quite straightforwardly, obtaining

$$
\begin{aligned}
\mathrm{M}\left\langle 0\left|r_{j, \kappa}^{\dagger} r_{i, \kappa^{\prime}}\right| 0\right\rangle_{\mathrm{M}} & =\frac{1}{e^{2 \pi \Omega}+1} \delta_{i j} \delta^{3}\left(\kappa-\kappa^{\prime}\right) \\
& \equiv \frac{1}{e^{\beta E}+1} \delta_{i j} \delta^{3}\left(\kappa-\kappa^{\prime}\right)
\end{aligned}
$$

where in the last step we have introduced the Rindler proper energy $E=a \Omega$ and the inverse Unruh temperature $\beta=$ $1 / T_{\mathrm{U}}$. Clearly, the same result would be obtained by considering the vacuum expectation value of $l_{j, \kappa}^{\dagger} l_{i, \kappa^{\prime}}$, which represents the particle number operator for an accelerated observer in $L$. Thus, from Eq. (37) we conclude that the Minkowski vacuum appears as a thermal state for the Rindler observer. The spectrum of particle is distributed according to the Fermi-Dirac statistics with temperature proportional to 
the magnitude of the acceleration. This is the well-known Unruh effect [1].

A comment is in order here: the spectrum (37) diverges for $\kappa=\kappa^{\prime}$. This is due to the fact the states created by the action of $r_{j, \kappa}^{\dagger}$ and $a_{\mathbf{k}}^{r, \dagger}$ on the vacuum are ill-defined, in the sense that they are not properly normalizable. To avoid unphysical divergences, a wavepacket based approach should be performed. This has been done explicitly for bosons in [34,54], where it has been shown that the ensuing spectrum retains the same profile as in the planewave formalism, with the Dirac delta function in energy-momentum being now replaced by the Kronecker delta. However, since wavepackets do not affect the spinorial structure of the field, a similar result is expected to be obtained for fermions as well. Then, in the wavepacket approach we can formally write

$\mathrm{M}\left\langle 0\left|r_{j, \kappa}^{\dagger} r_{i, \kappa^{\prime}}\right| 0\right\rangle_{\mathrm{M}}=\frac{1}{e^{2 \pi \Omega}+1} \delta_{i j} \delta_{\kappa \kappa^{\prime}}$,

without any pathological behavior for fixed $\kappa=\kappa^{\prime}$. In the next Section, we shall go on working with planewaves and only at the end we take care of this subtlety.

\section{Flavor mixing in Rindler spacetime}

Quantum mixing is among the most challenging and important topics in Particle Physics. In the well-established quantum mechanical version, it consists in the superposition of two states of particles with different masses, rotated each other of an angle $\theta$ to give rise to the so-called flavor states. Since its prediction by Pontecorvo [55], the theoretical basis of flavor mixing, and in particular of neutrino mixing, have been largely investigated [56], leading to the discovery of the phenomenon of neutrino oscillations. Experimental developments have later provided convincing evidences supporting Pontecorvo's proposal [57-60]. This has inevitably opened up new scenarios in the physics beyond the Standard Model, which account for the neutrino having a non-vanishing mass.

Two decades later Pontecorvo's quantum mechanical approach, a field theoretical formalism for mixed fields has been developed [32]. This has shown the shortcomings of the original description by pointing out the orthogonality between the vacuum for fields with definite flavors and that for fields with definite masses. Remarkably, this analysis has revealed a rich structure of the interacting-field vacuum as a $s u$ (2) coherent state, which in turn underlies the unitary inequivalence between the flavor and mass Fock spaces and the consequent alteration of the oscillation formula to include the antiparticle degrees of freedom (see Appendix A for more details).

All of the above field theoretical considerations on flavor mixing have been carried out in Minkowski background. Recently, they have been generalized to Rindler metric for the case of boson mixing [30]. To figure out how the flavor/mass inequivalence appears to the Rindler observer, let us consider the mixing transformations for Dirac fields in a simplified two-flavor model

$$
\begin{aligned}
& \Psi_{e}(t, \mathbf{x})=\cos \theta \Psi_{1}(t, \mathbf{x})+\sin \theta \Psi_{2}(t, \mathbf{x}), \\
& \Psi_{\mu}(t, \mathbf{x})=-\sin \theta \Psi_{1}(t, \mathbf{x})+\cos \theta \Psi_{2}(t, \mathbf{x}),
\end{aligned}
$$

where $\Psi_{e}, \Psi_{\mu}$ are the interacting fields with definite flavors (electron and muon), while $\Psi_{1}, \Psi_{2}$ denote the free fields with definite masses $m_{1}$ and $m_{2}$ (with $m_{2}>m_{1}$ ), satisfying the canonical anticommutator (13).

By assuming the standard planewave expansion (9) for $\Psi_{1}, \Psi_{2}$, Eqs. (39) take the form [32]

$$
\begin{gathered}
\Psi_{\ell}(t, \mathbf{x})=\sum_{r=1,2} \int d^{3} k N\left[a_{\mathbf{k}, \ell}^{r}(\theta, t) u_{\mathbf{k}, \sigma}^{r}(t) e^{i \mathbf{k} \cdot \mathbf{x}}\right. \\
\left.+b_{\mathbf{k}, \ell}^{r \dagger}(\theta, t) v_{\mathbf{k}, \sigma}^{r}(t) e^{-i \mathbf{k} \cdot \mathbf{x}}\right] \\
(\ell, \sigma)=\{(e, 1),(\mu, 2)\},
\end{gathered}
$$

where $\ell(\sigma)$ is the flavor (mass) index and we have rewritten the expansion (9) in a formally different (but equivalent) way by embedding the time-dependent exponential factor in the spinors $u_{\mathbf{k}, \sigma}^{r}(t)=u_{\mathbf{k}, \sigma}^{r} e^{-i \omega_{\mathbf{k}, \sigma} t}, v_{\mathbf{k}, \sigma}^{r}(t)=v_{\mathbf{k}, \sigma}^{r} e^{i \omega_{\mathbf{k}, \sigma} t}$. The flavor annihilators $a_{\mathbf{k}, \ell}^{r}(\theta, t)$ and $b_{\mathbf{k}, \ell}^{r}(\theta, t)$ have been computed explicitly in Appendix A. Furthermore, the algebraic nature of Eq. (39) has been explored in Eq. (A1), showing that each of them exhibits the structure of a rotation nested into a Bogoliubov transformation when considered at the level of ladder operators. As remarked above, the most striking implication of this peculiar structure turns out to be the inequivalence between the mass vacuum $|0\rangle_{1,2}=|0\rangle_{1} \otimes|0\rangle_{2}$ (such that $a_{\mathbf{k}, i}^{r}|0\rangle_{1,2}=b_{\mathbf{k}, i}^{r}|0\rangle_{1,2}=0, i=1,2$ ) and the flavor vacuum $|0(\theta, t)\rangle_{e, \mu}$ (such that $a_{\mathbf{k}, \ell}^{r}(\theta, t)|0(\theta, t)\rangle_{e, \mu}=$ $\left.b_{\mathbf{k}, \ell}^{r}(\theta, t)|0(\theta, t)\rangle_{e, \mu}=0, \forall t, \ell=e, \mu\right)$, which becomes a condensate of entangled massive particle/antiparticle pairs of density given by Eq. (A21).

Now, in the previous Section we have seen that a useful tool to quantize the Dirac field for an accelerated observer is the boost-mode expansion (28). Thus, to extend the QFT formalism of mixing to the Rindler metric, we first consider the analogue of Eq. (28) for flavor fields. By using the same approach as that adopted for the derivation of Eq. (40), we infer the following relation

$$
\begin{aligned}
\Psi_{\ell}(t, \mathbf{x})= & \sum_{j=1,2} \int d^{3} \kappa\left[c_{j, \kappa, \ell}(\theta, t) \psi_{j, \kappa, \sigma}^{-}(t, \mathbf{x})\right. \\
+ & \left.d_{j, \kappa, \ell}^{\dagger}(\theta, t) \psi_{j, \kappa, \sigma}^{+}(t, \mathbf{x})\right] \\
& (\ell, \sigma)=\{(e, 1),(\mu, 2)\}
\end{aligned}
$$

where the flavor ladder operators $c_{j, \kappa, \ell}$ and $d_{j, \kappa, \ell}$ are related to the flavor operators $a_{\mathbf{k}, \ell}^{r}$ and $b_{\mathbf{k}, \ell}^{r}$ in the planewave expansion (40) by 


$$
\begin{aligned}
c_{j, \kappa, \ell}(\theta, t) & =\sum_{r=1,2} \int d k_{x} F_{j, r, \sigma}\left(k_{x}, \Omega\right) a_{\mathbf{k}, \ell}^{r}(\theta, t), \quad(\ell, \sigma) \\
& =\{(e, 1),(\mu, 2)\},
\end{aligned}
$$

with $F_{j, r, \sigma}$ being given in Eq. (30), and

$$
\begin{aligned}
d_{j, \kappa \ell}^{\dagger}(\theta, t) & =\sum_{r=1,2} \int d k_{x} G_{j, r, \sigma}\left(k_{x}, \Omega\right) b_{\mathbf{k}, \ell}^{r \dagger}(\theta, t), \quad(\ell, \sigma) \\
& =\{(e, 1),(\mu, 2)\},
\end{aligned}
$$

with $G_{j, r, \sigma}$ being given in Eq. (32) (see Eqs. (29) and (31) for the corresponding relations in the mass basis). By relying on Eq. (33) and the related discussion, we stress that Eqs. (42) and (43) are canonical transformations which leave the flavor vacuum $|0(\theta, t)\rangle_{e, \mu}$ unchanged.

The expression (41) provides us with the springboard we were looking for. Indeed, we can closely follow the Rindler quantization scheme developed for massive fields at the end of Sect. (2) and apply to flavor fields. In doing so, we arrive at the following expansion

$$
\begin{aligned}
\psi_{\ell}(t, \mathbf{x})= & \sum_{j=1,2} \int d^{3} \kappa\left[r_{j, \kappa, \ell}(\theta, t) \mathcal{R}_{j, \kappa, \sigma}(t, \mathbf{x})\right. \\
& +r_{j, \kappa, \ell}^{\dagger}(\theta, t) \mathcal{R}_{j, \tilde{\kappa}, \sigma}(t, \mathbf{x}) \\
& +l_{j, \kappa, \ell}(\theta, t) \mathcal{L}_{j, \tilde{\kappa}, \sigma}(t, \mathbf{x}) \\
& \left.+l_{j, \kappa, \ell}^{\dagger}(\theta, t) \mathcal{L}_{j, \kappa, \sigma}(t, \mathbf{x})\right],
\end{aligned}
$$

where $(\ell, \sigma)=\{(e, 1),(\mu, 2)\}$, as usual. The flavor ladder operators for the Rindler observer read

$$
\begin{aligned}
& r_{j, \kappa, \ell}(\theta, t)=\frac{c_{j, \kappa, \ell}(\theta, t) e^{\frac{\pi \Omega}{2}}+d_{j, \kappa, \ell}^{\dagger}(\theta, t) e^{-\frac{\pi \Omega}{2}}}{\sqrt{2 \cosh (\pi \Omega)}}, \\
& l_{j, \kappa, \ell}^{\dagger}(\theta, t)=\frac{c_{j, \kappa, \ell}(\theta, t) e^{-\frac{\pi \Omega}{2}}-d_{j, \kappa, \ell}^{\dagger}(\theta, t) e^{\frac{\pi \Omega}{2}}}{\sqrt{2 \cosh (\pi \Omega)}},
\end{aligned}
$$

which have indeed the same structure as the transformations (36), the mass index being now replaced by the flavor one.

Two comments deserve attention here: first, as opposed to the Minkowski frequencies $\omega_{\mathbf{k}, i}, i=1,2$, the Rindler frequency $\Omega$ does not carry any index since it is independent of the field masses. Furthermore, we emphasize that the flavor ladder operators for the Rindler observer are given by the combinations of two Bogoliubov transformations, the thermal one responsible for the Unruh effect and encoded by the $\Omega$-dependent Bogoliubov coefficients, the second one arising from mixing and implicitly contained in $c_{j, \kappa, \ell}$ and $d_{j, \kappa, \ell}$ trough Eqs. (42) and (43). This is the same structure found for the case of boson field mixing in Rindler space (see $[30,61,62])$.
By using Eqs. (A11) and (A12), we can now explicitly write down $r_{j, \kappa, \ell}$ as

$$
\begin{aligned}
& r_{j, \kappa, \ell}(\theta, t)=\frac{1}{\sqrt{2 \cosh (\pi \Omega)}} \\
& \sum_{r=1,2} \int d k_{x}\left\{e^{\frac{\pi \Omega}{2}} F_{j, r, \sigma}\left(k_{x}, \Omega\right) a_{\mathbf{k}, \ell}^{r}(\theta, t)\right. \\
& \left.\quad+e^{-\frac{\pi \Omega}{2}} G_{j, r, \sigma}\left(k_{x}, \Omega\right) b_{\mathbf{k}, \ell}^{r \dagger}(\theta, t)\right\}, \\
& =\frac{1}{\sqrt{2 \cosh (\pi \Omega)}} \sum_{r=1,2} \int d k_{x}\left\{e^{\frac{\pi \Omega}{2}} F_{j, r, \sigma}\left(k_{x}, \Omega\right)\right. \\
& \quad\left\{\cos \theta a_{\mathbf{k}, 1}^{r}+\sin \theta \sum_{s=1,2}\left[\left(u_{\mathbf{k}, 1}^{r \dagger}(t), u_{\mathbf{k}, 2}^{s}(t)\right) a_{\mathbf{k}, 2}^{s}\right.\right. \\
& \left.\left.\quad+\left(u_{\mathbf{k}, 1}^{r \dagger}(t), v_{-\mathbf{k}, 2}^{s}(t)\right) b_{-\mathbf{k}, 2}^{s \dagger}\right]\right\} \\
& \quad+e^{-\frac{\pi \Omega}{2}} G_{j, r, \sigma}\left(k_{x}, \Omega\right)\left\{\cos \theta b_{\mathbf{k}, 1}^{r \dagger}\right. \\
& \quad+\sin \theta \sum_{s=1,2}\left[\left(v_{\mathbf{k}, 2}^{s \dagger}(t), v_{\mathbf{k}, 1}^{r}(t)\right)^{*} b_{\mathbf{k}, 2}^{s \dagger}\right. \\
& \left.\left.\left.\quad+\left(u_{-\mathbf{k}, 2}^{s \dagger}(t), v_{\mathbf{k}, 1}^{r}(t)\right)^{*} a_{-\mathbf{k}, 2}^{s}\right]\right\}\right\},
\end{aligned}
$$

and similarly for $l_{j, \kappa, \ell}$.

As in Eq. (37), we can now derive the vacuum distribution of mixed particles for the Rindler observer by computing the expectation value ${ }_{1,2}\left\langle 0\left|r_{j, \kappa, \ell}^{\dagger}(\theta, t) r_{j, \kappa, \ell}(\theta, t)\right| 0\right\rangle_{1,2}$. However, this faces us with the computation of nontrivial integrals, which cannot be solved analytically. Despite such technicalities, interesting implications can still be derived by setting $t=\eta=0$ and in the realistic approximation of relativistic neutrinos, i.e. $\epsilon \equiv \sqrt{m_{2}^{2}-m_{1}^{2}} / \omega_{\mathbf{k}, 1} \ll 1$. Under these assumptions, it is a matter of direct calculations to show that

$$
\begin{aligned}
& r_{j, \kappa, \ell}(\theta)|0\rangle_{1,2}=\frac{e^{\frac{\pi \Omega}{2}}}{\sqrt{2 \cosh (\pi \Omega)}} \\
& \sum_{r=1,2} \int d k_{x} F_{j, r, \sigma}\left(k_{x}, \Omega\right) \sin \theta\left(V_{\mathbf{k}}^{r 1} b_{-\mathbf{k}, 2}^{1 \dagger}\right. \\
& \left.+V_{\mathbf{k}}^{r 2} b_{-\mathbf{k}, 2}^{2 \dagger}\right)|0\rangle_{1,2} \\
& +\frac{e^{-\frac{\pi \Omega}{2}}}{\sqrt{2 \cosh (\pi \Omega)}} \sum_{r=1,2} \int d k_{x} G_{j, r, \sigma}\left(k_{x}, \Omega\right) \\
& \left(\cos \theta b_{\mathbf{k}, 1}^{r \dagger}+\sin \theta U_{\mathbf{k}} b_{\mathbf{k}, 2}^{r \dagger}\right)|0\rangle_{1,2},
\end{aligned}
$$

where the $U_{\mathbf{k}}$ and $V_{\mathbf{k}}$ coefficients have been defined in Eqs. (A13)-(A17). We stress that all the time-dependent quantities in the above equation must be intended as evaluated for $t=\eta=0$. 
Therefore, the particle spectrum at $t=\eta=0$ becomes

$$
\begin{aligned}
1,2 & \left\langle 0\left|r_{j, \kappa, \ell}^{\dagger}(\theta) r_{j, \kappa, \ell}(\theta)\right| 0\right\rangle_{1,2} \\
= & \frac{1}{e^{2 \pi \Omega}+1} \\
& +\frac{\sin ^{2} \theta}{2 \cosh (\pi \Omega)}\left[e^{\pi \Omega} \mathcal{N}_{j, \kappa, \sigma}^{F F}-e^{-\pi \Omega} \mathcal{N}_{j, \kappa, \sigma}^{G G}\right. \\
& \left.+\left(\mathcal{N}_{j, \kappa, \sigma}^{F G}+\text { c.c. }\right)\right],
\end{aligned}
$$

where the first contribution is the standard Fermi-Dirac distribution, while the terms multiplied by $\sin ^{2} \theta$ are the mixinginduced corrections. Here, we have used the relation (A20) for the Bogoliubov coefficients $U_{\mathbf{k}}$ and $V_{\mathbf{k}}$. Furthermore, we have implicitly taken into account the advantages of the wavepacket approach, which allows us to get rid of any pathological divergency in the evaluation of the spectrum for fixed momentum $\kappa$ (see the discussion at the end of Sect. 2).

The explicit expressions of $\mathcal{N}_{j, \kappa, \sigma}^{F F}, \mathcal{N}_{j, \kappa, \sigma}^{G G}$ and $\mathcal{N}_{j, \kappa, \sigma}^{F G}$ are rather awkward to exhibit. Here we provide their integral expressions

$$
\begin{aligned}
\mathcal{N}_{j, \kappa, \sigma}^{F F} \equiv & \sum_{r=1,2} \int d k_{x}\left|F_{j, r, \sigma}\left(k_{x}, \Omega\right)\right|^{2}\left|V_{\mathbf{k}}\right|^{2} \\
\mathcal{N}_{j, \kappa, \sigma}^{G G} \equiv & \sum_{r=1,2} \int d k_{x}\left|G_{j, r, \sigma}\left(k_{x}, \Omega\right)\right|^{2}\left|V_{\mathbf{k}}\right|^{2} \\
\mathcal{N}_{j, \kappa, \sigma}^{F G} \equiv & \sum_{r=1,2} \int d k_{x} F_{j, r, \sigma}\left(k_{x}, \Omega\right)\left[G_{j, 1, \sigma}^{*}\left(-k_{x}, \Omega\right) V_{\mathbf{k}}^{r 1}\right. \\
& \left.+G_{j, 2, \sigma}^{*}\left(-k_{x}, \Omega\right) V_{\mathbf{k}}^{r 2}\right] U_{\mathbf{k}} .
\end{aligned}
$$

For concreteness, we consider the case of spin $j=1$ and flavor $\ell=e$ in Eq. (48) (the same considerations hold true for $j=2$ and/or $\ell=\mu$ ). In turn, this amounts to setting the mass index $\sigma$ equal to one. Then, by replacing Eqs. (30), (32) and (A19) into (49) and (50) and rewriting

$m_{2}=\sqrt{m_{1}^{2}+\epsilon^{2} \omega_{\mathbf{k}, 1}^{2}}, \quad \omega_{\mathbf{k}, 2}=\omega_{\mathbf{k}, 1} \sqrt{1+\epsilon^{2}}$,

one can show that

$\mathcal{N}_{1, \kappa, 1}^{F F} \sim \mathcal{O}\left(\epsilon^{4}\right), \quad \mathcal{N}_{1, \kappa, 1}^{G G} \sim \mathcal{O}\left(\epsilon^{4}\right)$.

On the other hand, from Eq. (51) it follows that

$\mathcal{N}_{1, \kappa, 1}^{F G} \simeq \int d k_{x} \epsilon^{2} \mathcal{I}_{\kappa}\left(k_{x}\right)$,

where the function $\mathcal{I}_{\kappa}\left(k_{x}\right)$ is explicitly given in Appendix B. By further manipulation, we finally arrive at

$\mathcal{N}_{1, \kappa, 1}^{F G} \simeq \frac{\left|\Delta m^{2}\right|}{\mu_{\vec{k}, 1}^{2}} e^{-\pi \Omega} \mathcal{H}\left(\mu_{\vec{k}, 1}\right)$,

where we have factorized out the dependence on both $\left|\Delta m^{2}\right|=\left|m_{2}^{2}-m_{1}^{2}\right|$ and $\Omega$, and $\mathcal{H}\left(\mu_{\vec{k}, 1}\right)$ denotes the (dimensionless) $k_{x}$-integral of the residual function. Although such integral cannot be evaluated analytically, numerical estimations show that it assumes finite values and its real part is positive, at least in the regime of interest (see the discussion below Eq. (B4)).

Thus, to the leading order in $\epsilon$ we can neglect the corrections $\mathcal{N}_{1, \kappa, 1}^{F G}$ and $\mathcal{N}_{1, \kappa, 1}^{G G}$ in Eq. (48) and retain only $\mathcal{N}_{1, \kappa, 1}^{F G}$. In this way the modified Unruh spectrum takes the form

$$
\begin{aligned}
& 1,2\left\langle 0\left|r_{1, \kappa, e}^{\dagger}(\theta) r_{1, \kappa, e}(\theta)\right| 0\right\rangle_{1,2} \\
& \simeq \frac{1}{e^{2 \pi \Omega}+1} \\
& \quad+\frac{\left|\Delta m^{2}\right|}{\mu_{\vec{k}, 1}^{2}} \sin ^{2} \theta \operatorname{Re}\left\{\mathcal{H}\left(\mu_{\vec{k}, 1}\right)\right\} \frac{e^{-\pi \Omega}}{\cosh (\pi \Omega)}
\end{aligned}
$$

where $\operatorname{Re}\{z\}$ indicates the real part of $z$. Notice that this has the same structure as the modified spectrum found for mixing of bosons [30]. Furthermore, the mixing-induced correction tends to $\left|\Delta m^{2}\right| / \mu_{\vec{k}, 1}^{2} \sin ^{2} \theta \operatorname{Re}\left\{\mathcal{H}\left(\mu_{\vec{k}, 1}\right)\right\} \ll 1$ for $\Omega \rightarrow 0$, while it vanishes for large $\Omega$.

Within the framework of Boltzmann-Gibbs thermodynamics, the result (48) (or equivalently (56)) is a signature of the broken thermality of Unruh radiation for mixed fields. Such an effect has been discussed in more detail in [30] for the case of boson mixing. Regardless of the specific nature of the fields, it can essentially be traced back to the complex nature of the quantum vacuum for mixed fields. Indeed, whilst in the absence of mixing this state is made up of one type of pairwise correlated particles/antiparticles, for mixed fields it contains pairs of both equal and different types (see the discussion in Appendix A). This gives rise to a nontrivial modification of the Unruh distribution, which however is recovered for $\theta=0$ and/or $m_{2}=m_{1}$, consistently with the vanishing of mixing. We further elaborate on the physical meaning of Eq. (56) in the next section, where we revisit the above effect in the context of nonextensive Tsallis theory.

\section{4 q-generalized Tsallis statistics for neutrino mixing}

In Boltzmann-Gibbs statistical mechanics, entropy is as a measure of the number of possible microscopic states of a system in thermodynamic equilibrium, consistent with its macroscopic thermodynamic properties. This definition is enclosed in the well-known formula

$S_{\mathrm{BG}}=-\sum_{i=1}^{W} p_{i} \log p_{i}$

for a set of $W$ discrete microstates, where $p_{i}$ is the probability of the $i$-th microscopic configuration with the condition $\sum_{i=1}^{W} p_{i}=1$. Clearly, if probabilities are all equal, this takes the form $S_{\mathrm{BG}}=\log W$. 
It is immediate to check that the entropy as defined above is additive, which means that, given two probabilistically independent systems $A$ and $B$ with entropies $S_{\mathrm{BG}}(A)$ and $S_{\mathrm{BG}}(B)$, the total entropy is $S_{\mathrm{BG}}(A+B)=S_{\mathrm{BG}}(A)+$ $S_{\mathrm{BG}}(B)$. Intuitively, this reflects the fact that, in the absence of interactions, the number of total states of a composite systems is simply given by the sum of the states of its constituent parts.

Although Boltzmann-Gibbs statistics is the correct way of approaching thermodynamic ergodic systems, it has been argued that strong correlations may be sometime responsible for the emergence of exotic features that lye outside the domain of description of the standard theory. The most emblematic example of systems falling within this category are the gravitational systems, as already pointed out by Gibbs at the beginning of the last century. Hence, in such cases a generalization of Boltzmann-Gibbs statistics and the related additive entropy is unavoidable.

Along this line, in [35-38] it has been shown that a proper generalization of Eq. (57) for complex systems exhibiting long-range interactions and/or spacetime entanglement is given by the following nonadditive entropy

$S_{q}=\frac{1-\sum_{i=1}^{W} p_{i}^{q}}{q-1}=\sum_{i=1}^{W} p_{i} \log _{q} \frac{1}{p_{i}}, \quad q \in \mathbb{R}^{+}$,

where

$\log _{q} z \equiv \frac{z^{1-q}-1}{1-q}, \quad\left(\log _{1} z=\log z\right)$.

This is the so-called $q$-Tsallis entropy and the framework built upon it is the nonextensive (or Tsallis) statistical mechanics. We notice that the Boltzmann-Gibbs definition (57) is a special case of $S_{q}$ for $q \rightarrow 1$. Moreover, by considering again two independent systems such that $p_{i j}^{A+B}=p_{i}^{A} p_{j}^{B}, \forall(i, j)$, Eq. (58) yields

$S_{q}(A+B)=S_{q}(A)+S_{q}(B)+(1-q) S_{q}(A) S_{q}(B)$,

which shows that $S_{q}$ is superadditive or subadditive, depending on whether $q<1$ or $q>1$. Since the $q$-entropic index quantifies the departure from Boltzmann-Gibbs, it is typically named nonextensive Tsallis parameter. As remarked above, the definition (58) works quite well for strongly gravitating systems, and in particular for black holes [37], although in recent years encouraging results have been obtained in the study of the cosmic background radiation [44,45], lowdimensional dissipative systems [38], solar neutrinos [46] and polymer chains [47], among others.

The $q$-generalized entropy (58) naturally leads to nontrivial modifications of the standard tools of Boltzmann-Gibbs theory. For instance, in [63] it has been discussed how the removal of the assumption of extensivity of entropy from statistical mechanics affects the core thermodynamic rela- tions, including the zero-th law of thermodynamics and the concepts of temperature and pressure. Similarly, in [64-68] Tsallis entropy has been used to derive the following generalized average occupational numbers

$N_{q}(E)=\frac{1}{[1+(q-1) \beta E]^{1 /(q-1)} \pm 1}$,

which are obtained through the usual procedure of maximizing Tsallis entropy under the constraints of keeping constant the average internal energy and the average number of particles. Here the upper (lower) sign refers to the generalized Fermi-Dirac (Bose-Einstein) distribution. ${ }^{2}$ Furthermore $\beta=1 / T$, where $T$ is the temperature of the system. We remark that the usual Fermi-Dirac and Bose-Einstein distributions are recovered in the $q \rightarrow 1$ limit.

To make physical sense, the distributions (61) must be non-negative and real-valued. This gives rise to the following constraint

$$
\left\{\begin{array}{l}
0 \leq E \leq[(1-q) \beta]^{-1} \text { for } q<1, \\
E \geq 0 \text { for } q>1
\end{array}\right.
$$

Following [70], we also point out that Eq. (61) only provides a preliminary but still useful approximation. Indeed, one cannot derive the exact analytical expression of the generalized distribution for arbitrary values of $q$, due to the nonseparability of the partition function. Nevertheless, the error committed turns out to be fairly negligible at very low temperatures for systems with large numbers of particles, as it is for quantum fields (see [70] for more detailed numerical estimations). Accordingly, since the Unruh temperature is extremely small even for huge accelerations, ${ }^{3}$ the use of Eq. (61) is well justified.

In the previous section we have emphasized that the Unruh spectrum for mixed fields loses its typical thermal profile owing to the entangled structure acquired by the quantum vacuum, which becomes a (time-dependent) $s u(2)$ coherent state (see Eqs. (48) or (56)). Based on the observation that the generalized Tsallis framework well describes strongly correlated systems, either on quantum or classical grounds, in [34] the question arose of how such a result could appear when rephrased in the language of nonextensive Tsallis statistics. Remarkably, for mixing of bosons it has been shown that the modified Unruh distribution can be mapped into the $q$ generalized Bose-Einstein distribution (61), provided that the $q$-entropic index is properly related to the characteristic mixing parameters $\sin \theta$ and $\Delta m$.

\footnotetext{
${ }^{2}$ With regard to the $q$-generalized Fermi-Dirac distribution, it is worth noting that apparent violations of Pauli's principle arising in the nonextensive quantum thermostatistics at finite temperature have been discussed in [69] in connection with the possible existence of dark (à la Bacry) fermions.

${ }^{3}$ Let us remind that $T_{\mathrm{U}}$ is smaller than $1 \mathrm{~K}$ even for accelerations up to $10^{21} \mathrm{~m} / \mathrm{s}^{2}$.
} 
Following this analysis, here we extend the Tsallis paradigm to mixing of fermions as well. Clearly, since the correction in Eq. (56) slightly affects the Fermi-Dirac spectrum at both high and low energy regimes, it is reasonable to expand the generalized distribution (61) (with the + sign in the denominator) for tiny departures of $q$ from unity. To the leading order, we then obtain

$$
\begin{aligned}
N_{q}(E) & \simeq \frac{1}{e^{\beta E}+1}+\frac{(\beta E)^{2}}{4[1+\cosh (\beta E)]}(q-1), \\
& =\frac{1}{e^{\beta E}+1}+\frac{1}{8}(\beta E)^{2} \operatorname{sech}^{2}\left(\frac{\beta E}{2}\right)(q-1),
\end{aligned}
$$

to be compared with the corresponding Tsallis-induced correction for bosons (see Eq. (39) of [34])

$N_{q}(E) \simeq \frac{1}{e^{\beta E}-1}+\frac{1}{8}(\beta E)^{2} \operatorname{csch}^{2}\left(\frac{\beta E}{2}\right)(q-1)$.

In passing, we note that a similar perturbative analysis in nonextensive statistics has been carried out in the context of high-energy heavy-ion collisions [71], starting from the experimental evidence that high-energy nucleus-nucleus collisions cannot be described in terms of superpositions of elementary nucleon-nucleon interactions. In that case, the use of Tsallis statistics is motivated by the hypothesis that collective effects in the hadronic medium can be traced back to some kind of memory effects and long-range forces that may occur during high-energy ion collisions.

Now, Eq. (63) can be compared to the modified spectrum (56) by setting $E=a \Omega$ (the proper energy of Rindler modes) and $\beta=1 / T_{\mathrm{U}}=2 \pi / a$. Straightforward calculations lead to

$N_{q}(\Omega) \simeq \frac{1}{e^{2 \pi \Omega}+1}+\frac{\pi^{2}}{2} \Omega^{2} \operatorname{sech}^{2}(\pi \Omega)(q-1)$.

Although we are only retaining the first term in the expansion, we notice that the energy dependence of the $q$-generalized spectrum gets nontrivially altered.

Let us now investigate the correspondence between the modification of Unruh distribution arising in the context of flavor mixing and that obtained in the nonextensive Tsallis theory, respectively. By equating Eqs. (56) and (65), we infer

$$
\begin{aligned}
q & =1+\mathcal{F}_{\theta}\left(\Delta m^{2}, \mu_{\vec{k}, 1}\right) \frac{1+e^{-2 \pi \Omega}}{\Omega^{2}} \\
& \simeq 1+\frac{\mathcal{F}_{\theta}\left(\Delta m^{2}, \mu_{\vec{k}, 1}\right)}{\Omega^{2}},
\end{aligned}
$$

where in the last step we have approximated

$\frac{1+e^{-2 \pi \Omega}}{\Omega^{2}} \simeq \frac{1}{\Omega^{2}}, \quad$ for $\Omega>0$,

apart from an irrelevant factor, and we have defined
$\mathcal{F}_{\theta}\left(\Delta m^{2}, \mu_{\vec{k}, 1}\right) \equiv \frac{\left|\Delta m^{2}\right| \operatorname{Re}\left\{\mathcal{H}\left(\mu_{\vec{k}, 1}\right)\right\}}{\pi^{2} \mu_{k, 1}^{2}} \sin ^{2} \theta$.

The function $\mathcal{H}\left(\mu_{\vec{k}, 1}\right)$ is defined in Eqs. (B2)-(B4) of Appendix B.

Now, since $\operatorname{Re}\left\{\mathcal{H}\left(\mu_{\vec{k}, 1}\right)\right\}>0$ (at least in the regime we are considering, see Appendix B), from the above relations we infer that $q>1$, indicating the subadditive regime of Tsallis entropy, according to Eq. (60). Furthermore, from Eq. (62) we see that there is no upper limit on the energy scale. By contrast, for the case of boson mixing one has $q<1$, which corresponds to a flavor vacuum entropy higher than the sum of the entropies of the two mass vacua taken separately (Tsallis superadditive regime) [34].

Therefore, in the above effective picture, the properties of Unruh condensate for mixed particles can be described in terms of the nonextensive Tsallis statistics, with the $q$ entropic index satisfying the condition (66). As expected, the departure of $q$ from unity depends on the mixing angle and the mass difference in such a way that the usual BoltzmannGibbs theory with $q=1$ is recovered for $\theta=0$ and/or $\Delta m=0$, consistently with the vanishing of mixing in both cases. The same behavior occurs in the ultrarelativistic limit $\Delta m / \mu_{\vec{k}, 1} \rightarrow 0$, since $\operatorname{Re}\left\{\mathcal{H}\left(\mu_{\vec{k}, 1}\right)\right\}$ is finite even for large momenta. This can be understood by observing that in such approximation the field theoretical description of flavor mixing reproduces the standard quantum mechanical framework, in which the flavor and mass representations are connected by a simple rotation, and thus the related Fock spaces are unitarily equivalent to each other (see the discussion in Appendix A).

Following [34], we now exploit Eq. (66) to provide an alternative interpretation for the result (56). Indeed, instead of regarding the mixing-induced distortion of the spectrum as a nonthermal effect within Boltzmann-Gibbs framework, we can trace its origin back to a modification of the vacuum distribution of particles/antiparticle pairs at a more fundamental statistical level. We remark that a similar connection between the Unruh effect and Tsallis statistics has been exhibited in $[72,73]$ in the context of deformed uncertainty relations accounting for a minimal length at Planck scale (Generalized Uncertainty Principle).

As for the case of bosons, the identification in Eq. (66) shows that the $q$-entropic index exhibits a running behavior as a function of the Rindler frequency $\Omega$. This is quite a common behavior for quantum field theoretical and quantum gravity systems. An analogous study in Tsallis statistics with a varying nonextensive parameter has been recently developed in cosmology in [48]. Interestingly, we notice that, while for mixing of bosons the deviation of $q$ from unity depends on $\Omega^{-1}$ [34], in the present case it scales as $\Omega^{-2}$. Of course, this does not entail any dimensional problem, being the Rindler frequency $\Omega$ dimensionless. 
In this connection, one may argue that Eq. (66) exhibits a pathological behavior for arbitrarily small frequencies, as it diverges in the $\Omega \rightarrow 0$ limit. However, as discussed in [34], this must be interpreted as a warning concerning the domain of validity of our approximation. Indeed, for $\Omega$ below a certain threshold $\Omega_{\min }$, it happens that the $q$-entropic index might largely deviate from unity, which a posteriori would invalidate the expansion (65). To keep the self-consistency of our analysis, we have to require that $|q-1| \ll 1$. In turn, this gives rise to the infrared frequency cutoff

$$
\begin{aligned}
\Omega \gg \Omega_{\min } & =\left[F_{\theta}\left(\Delta m^{2}, \mu_{\vec{k}, 1}\right)\right]^{1 / 2} \\
& =\left[\frac{\left|\Delta m^{2}\right| \operatorname{Re}\left\{\mathcal{H}\left(\mu_{\vec{k}, 1}\right)\right\}}{\pi^{2} \mu_{k, 1}^{2}} \sin ^{2} \theta\right]^{1 / 2},
\end{aligned}
$$

which means that the more accurate the approximation of relativistic neutrinos $\Delta m / \mu_{\vec{k}, 1} \ll 1$, the higher the number of Rindler modes that fit with the $q$-generalized FermiDirac distribution (61). Clearly, the entire frequency spectrum is spanned for $F_{\theta} \rightarrow 0$. To give some numerical estimations, here we notice that, by setting the sampling values $\theta=\pi / 4, \Delta m^{2} \sim 10^{-5} \mathrm{eV}^{2}$ and $k_{y}=k_{z} \simeq 10 \mathrm{GeV}$, we get $\Omega_{\text {min }} \simeq 10^{-8}$. On the other hand, the departure from extensivity becomes increasingly negligible for large $\Omega$. In particular the Boltzmann-Gibbs statistics with $q=1$ is recovered for $\Omega \rightarrow \infty$. As explained in [34], this is somehow consistent with the fact that the higher the energy of the level we are considering, the lower the expected number of particles it can accommodate, with both the Fermi-Dirac and $q$-generalized distributions approaching zero as $\Omega \rightarrow \infty$. Therefore, it is reasonable to expect that the difference between the two spectra narrows for relatively large $\Omega$. This is exactly the same behavior found in [34] for mixing of bosons.

The connection established between the modification (56) induced by flavor mixing and the $q$-generalized Fermi-Dirac distribution (63) can be explained in terms of the nontrivial structure acquired by the vacuum for mixed fields (see Appendix A). Indeed, due to the peculiar features of mixing transformations in quantum field theory, this state turns out to be a condensate of entangled particle/antiparticle pairs of both equal and different types. As a consequence, while the standard Unruh effect may be seen as due to single-type fluctuations spontaneously appearing close to the Rindler horizon (one component falling back into the horizon, the other escaping as Unruh radiation), in the presence of field mixing it can be generated by different types of virtual pairs, resulting in a deformed spectrum of Unruh radiation. This has been pictorially represented in Fig. 2 by associating different (online) colors to different types of particles. We have shown above that such effect can be framed in the context of Tsallis statistics, with a modified distribution given by the $q$-generalized distribution (63) based on nonadditive Tsallis

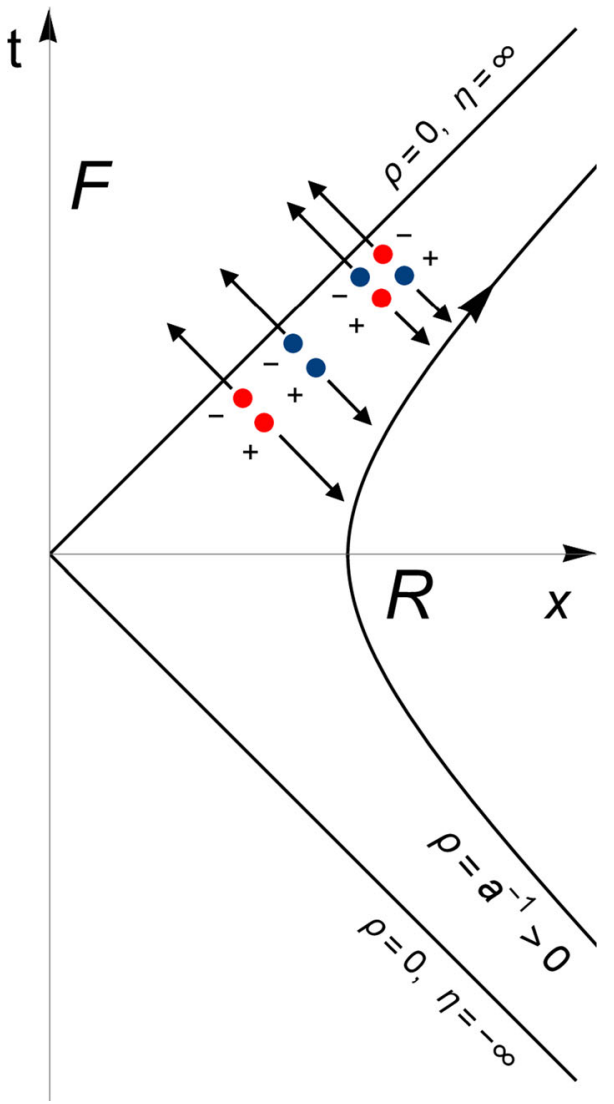

Fig. 2 Pictorial interpretation of the mixing-induced modification of Unruh condensate. Different (online) dot colors correspond to different types of pairwise correlated particles/antiparticles. Unruh effect originates from vacuum fluctuations close to Rindler horizon. For free (i.e unmixed) fields, the vacuum state is made up of single-type pairs (either blue-blue or red-red). By contrast, in the presence of field mixing its structure becomes far richer due to the simultaneous appearance of hybrid pairs (red-blue and blue-red). The ensuing spectrum can still be featured as a thermal-like bath, provided that vacuum constituents are assumed to be distributed according to nonextensive Tsallis statistics

entropy (58). In particular, the departure $q-1$ from extensivity is quantified by the characteristic mixing parameters and the energy scale (see Eq. (66)). As a remark, from Eq. (60) we also emphasize that $q>1$ implies that mixed particles are packaged in the Unruh condensate in such a way that the total entropy of the vacuum for mixed fields is lower than the sum of the vacuum entropies for the corresponding free fields. Heuristically speaking, this can be understood as follows: suppose we dispose of a detector of red-type particles only (analogous considerations hold for a detector of bluetype particles). In the absence of mixing, the detection of a particle definitely corresponds to the same type of antiparticle fallen back into the horizon. By contrast, for mixed fields, if we see a red particle, it may be associated to a redantiparticle, as well as to a blue-antiparticle crossing inward the horizon, with the sum of probabilities being normalized to unity (see Fig. 2). However, a blue-antiparticle that falls into the horizon can also lead to the escape of a blue-particle, 
which will remain undetected under our hypothesis. Since, for the Pauli exclusion principle, two identical fermions cannot occupy the same quantum state simultaneously, we infer that the existence of blue-antiparticle/blue particle pairs in the vacuum affects the microscopic configuration of the system by reducing the available phase space volume for the blueantiparticle/red particle pairs. Indeed, it may happen that the blue antiparticle of a blue-blue pair with given quantum numbers crosses inward the horizon before the blue antiparticle of a blue-red pair with the same quantum numbers does. Then, for its part the blue antiparticle of the blue-red pair will be prevented from entering the horizon and its companion from escaping as Unruh radiation. This results in a lower number of possible microstates for the observed system and a related decrease of the total entropy with respect to the case of unmixed fields. Of course, such a behavior does not occur for bosons, for which the condensate structure of vacuum made up of hybrid types of pairs, combined with the fact that bosons are not subject to the Pauli exclusion principle, leads to the opposite superadditive regime for Tsallis entropy [34].

It is interesting to observe that a similar comparative analysis of the generalized thermostatistic properties of bosons and fermions has been carried out in [74] in the context of the quantum $q$-deformed algebra. This differs from Tsallis framework in that it relies on the modification of the quantum algebra of the creation and annihilation operators based on the $q$-calculus [75], rather than the modification of the partitioning of the microstates of the many body system. In that case, it has been shown that $q$-deformed bosons and fermions have an enhancement of the quantum statistical effects compared to standard behavior. In addition, in [76] the thermodynamic geometry of an ideal q-deformed boson and fermion gas has been constructed, investigating some properties such as the stability and statistical interaction.

The above considerations allow us to state that the correlations induced by mixing do spoil the macroscopic properties of Unruh condensate by affecting the statistical behavior of its microscopic configurations. In this connection, we notice that nonextensive statistics based on Tsallis entropies, such as the relative entropy and the Peres criterion, have been widely used in the study of entanglement in the last years [77-79].

It would be interesting to explore whether this link between deviation from Boltzmann-Gibbs theory and field mixing manifests also within the framework of other extended statistics, such as Kaniadakis statistics coming from relativistic corrections to the Boltzmann theory [80,81]. Along this line, preliminary results could be derived by exploiting the existing relation between Tsallis and Kaniadakis entropic measures (see, for instance, [82]). What we can envisage at this stage is that, since Kaniadakis extension is a generalization of Boltzmann-Gibbs entropy that works in relativistic theory and given that mixing-induced effects on the vacuum are more relevant in the non-relativistic regime (see
Appendix A), corrections due to the Kaniadakis-like description might be even smaller (though non-vanishing) than those found in the present analysis. This study offers anyway inspiring inputs for future extension of our formalism.

\section{Conclusions and outlook}

The Unruh effect is a notorious prediction built in quantum field theory, which states that empty space appears as a thermal bath of particles from the point of view of an accelerating observer [1]. Recently, it has been shown that this effect is nontrivially spoilt when dealing with mixing of fields with different masses [30,31], leading to a nonthermal distortion of the vacuum condensate. Following the analysis of [34] for boson mixing, here we have revisited this phenomenon in the context of nonextensive Tsallis thermodynamics. By considering the superposition of two Dirac neutrino fields in the relativistic approximation, we have provided an effective description of the modified Unruh spectrum in terms of the $q$-generalized Fermi-Dirac distribution based on the nonadditive Tsallis entropy. In this framework, the departure from the standard Boltzmann-Gibbs theory turns out to be quantified by the mass difference of the mixed fields and the mixing angle. Furthermore, the condition $q>1$ indicates the subadditive feature of Tsallis entropy. In this sense, the present result differs from that of [34], where the opposite superadditive regime has been obtained. We wish to emphasize that our analysis is still valid for $q \rightarrow 1$ (BoltzmannGibbs limit). From Eqs. (66) and (68), it is clear that this limit occurs when the mixing angle $\theta$ and/or the mass difference $\Delta m^{2}$ vanish. In other terms, switching off the mixing (and the vacuum correlations it induces) amounts to recover the conventional extensive framework and, thus, the standard well-known properties of Unruh effect, as expected.

As remarked in [34], the above analysis provides us with a more conservative interpretation for the result of [30,31], since it allows us to extend the usual thermal behavior of Unruh effect to the case of mixed fields. In other terms, one can maintain a thermal-like picture for the vacuum condensate, provided that the distribution of particle/antiparticle pairs is assumed to obey Tsallis (rather than Boltzmann) prescription. In turn, the origin of this statistical effect can be ascribed to the nontrivial structure acquired by the vacuum for mixed fields, which becomes a hybrid condensate of entangled particle-antiparticle pairs. Therefore, in this scenario it is still possible to associate an effective temperature to the vacuum condensate, which however would be different from the standard definition (1), since it would depend on the entropic index (see [63] for more details). In particular, the new physical temperature should be generalized from $T=1 /\left(k_{B} \beta\right)$ ( $k_{B}$ being Boltzmann constant) to $T^{\prime}=1 /\left(k_{T} \beta^{*}\right)$, with both $k_{T}$ and $\beta^{*}$ depending on $q$ 
in such a way that, for $q$ approaching unity, $k_{T} \rightarrow k_{B}$ and $\beta^{*} \rightarrow \beta$, recovering the usual definition [63]. This would imply that the temperature $T$ appearing in Eq. (63) of the manuscript is not exactly Unruh temperature, but deviates by a $q$-dependent correction. In this sense, Unruh temperature for mixed fields may exhibit a more complicated expression than the usual one depending only on the observer's acceleration. We are currently working on this point and aim to provide more details in a future work.

In this respect, it should be emphasized that a $q$-dependent expression for Unruh temperature has been derived in the context of the generalized uncertainty principle (GUP) in $[72,73]$. In that case, the departure of Unruh effect from Boltzmann statistics is induced by the emergence of a minimal length of the order of Planck scale, which affects the phase-space structure by changing the elementary cell volume. In light of this similarity, it would be interesting to investigate whether there exists any kind of connection between the two frameworks. For instance, a suggestive idea could be to rethink the phenomenon of mixing as an effect induced by background geometry, just like the GUP arises from a modified geometry of spacetime at quantum gravity scale.

Apart from these long-term perspectives, the present analysis can be refined by looking at the following issues: to avoid technicalities, in our computations we have considered a simplified model involving only two fields. However, we expect that the generalization to the more realistic threeflavor scenario does not affect our results at the conceptual level. Furthermore, we have made use of the assumption of relativistic neutrinos and, at the same time, we have expanded the $q$-generalized Fermi-Dirac distribution for small deviations of $q$ from unity. To strengthen our outcome, the exact calculation would be required. Then, the question arises as to how Eq. (66) appears when relaxing any approximation. We also developed computations for $t=0$, neglecting the timedependence of the vacuum for mixed fields. Clearly, a more complete analysis should take into account the time evolution of the vacuum (and any possible deviation from thermal equilibrium) as well. A further direction would be to analyze the above framework by considering neutrinos as Majorana fields. As well-known, indeed, the very nature of neutrinos - Dirac or Majorana - is still unclear, the only practical way to discriminate being the search for neutrinoless double $\beta$ decays. Based on [83], where the Unruh effect was shown to be insensitive to the Majorana/Dirac dichotomy, also in this case we envisage no significant deviations from the current result.

Finally, it is easy to understand that experimental tests of our prediction are extremely challenging at the present, the reason being that Unruh radiation itself has not yet been detected. Nevertheless, promising proposals have been put forward in the context of analog models. Among these, we mention experiments on water waves [24,25], graphene [27] and other condensed matter systems $[26,28]$. Therefore, such models represent the only source to address experimentally the Unruh effect - and any possible deviation from the standard behavior - to date.

Acknowledgements One of the authors (GGL) is grateful to Costantino Tsallis (Centro Brasileiro de Pesquisas Fisicas, Brazil) for helpful conversations. The authors would like to thank the anonymous Referees for valuable comments that helped us to improve the quality of the manuscript.

Data Availability Statement This manuscript has no associated data or the data will not be deposited. [Authors' comment: Data sharing is not applicable to this article as no datasets were generated or analyzed during the current study.]

Open Access This article is licensed under a Creative Commons Attribution 4.0 International License, which permits use, sharing, adaptation, distribution and reproduction in any medium or format, as long as you give appropriate credit to the original author(s) and the source, provide a link to the Creative Commons licence, and indicate if changes were made. The images or other third party material in this article are included in the article's Creative Commons licence, unless indicated otherwise in a credit line to the material. If material is not included in the article's Creative Commons licence and your intended use is not permitted by statutory regulation or exceeds the permitted use, you will need to obtain permission directly from the copyright holder. To view a copy of this licence, visit http://creativecomm ons.org/licenses/by/4.0/.

Funded by $\mathrm{SCOAP}^{3}$.

\section{Appendix A: QFT of flavor mixing}

This Appendix contains technical details on the quantization of mixed Dirac fields in Minkowski spacetime [32]. To avoid technicalities and make the physical insight as transparent as possible, we focus on a toy model involving two fields only. However, the same considerations hold in the three-flavor case [84].

To better understand the origin of the genuinely QFT features of flavor mixing, let us rewrite the transformations (39) in terms of the algebraic generator $G_{\theta}(t)$ as [32]

$\Psi_{\ell}(t, \mathbf{x})=G_{\theta}^{-1}(t) \Psi_{\sigma}(t, \mathbf{x}) G_{\theta}(t)$,

where $(\ell, \sigma)=\{(e, 1),(\mu, 2)\}$ and

$$
\begin{aligned}
G_{\theta}(t)= & \exp \left\{\theta \int d ^ { 3 } x \left[\Psi_{1}^{\dagger}(t, \mathbf{x}) \Psi_{2}(t, \mathbf{x})\right.\right. \\
& \left.\left.-\Psi_{2}^{\dagger}(t, \mathbf{x}) \Psi_{1}(t, \mathbf{x})\right]\right\} .
\end{aligned}
$$

By introducing the operators

$S_{+} \equiv \int d^{3} x \Psi_{1}^{\dagger}(t, \mathbf{x}) \Psi_{2}(t, \mathbf{x}), \quad S_{-}=\left(S_{+}\right)^{\dagger}$,

$G_{\theta}$ can be cast in the equivalent form

$G_{\theta}(t)=\exp \left[\theta\left(S_{+}-S_{-}\right)\right]$. 
It is easy to verify that $G_{\theta}(t)$ belongs to the $s u(2)$ group, whose algebra is closed by

$$
\begin{aligned}
& S_{3}=\frac{1}{2} \int d^{3} x\left(\Psi_{1}^{\dagger}(t, \mathbf{x}) \Psi_{1}(t, \mathbf{x})-\Psi_{2}^{\dagger}(t, \mathbf{x}) \Psi_{2}(t, \mathbf{x})\right), \\
& S_{0}=\frac{1}{2} \int d^{3} x\left(\Psi_{1}^{\dagger}(t, \mathbf{x}) \Psi_{1}(t, \mathbf{x})+\Psi_{2}^{\dagger}(t, \mathbf{x}) \Psi_{2}(t, \mathbf{x})\right) .
\end{aligned}
$$

In the QFT treatment of flavor mixing, the generator $G_{\theta}(t)$ provides the dynamical map between the Fock space $\mathcal{H}_{e, \mu}$ for definite flavor fields and the Fock space $\mathcal{H}_{1,2}$ for definite mass fields. We shall refer to these spaces as flavor and mass Fock spaces, respectively [32]. Thus, we can write

$G_{\theta}^{-1}(t): \mathcal{H}_{1,2} \mapsto \mathcal{H}_{e, \mu}$,

Specifically, for the mass vacuum $|0\rangle_{1,2}=|0\rangle_{1} \otimes|0\rangle_{2}$ the above relations leads to

$|0(\theta, t)\rangle_{e, \mu}=G_{\theta}^{-1}(t)|0\rangle_{1,2}$,

where we have denoted by $|0(\theta, t)\rangle_{e, \mu}$ the vacuum for flavor fields.

The peculiar properties of QFT mixing must be sought in the nontrivial nature of $G_{\theta}$. Indeed, while for quantum mechanical systems this is a unitary operator preserving the canonical anticommutation relations, in QFT it turns out to be nonunitary in the infinite volume limit. From Eq. (A7), it follows that the vacua $|0\rangle_{1,2}$ and $|0(\theta, t)\rangle_{e, \mu}$ become orthogonal to each other, resulting in unitarily (i.e. physically) inequivalent mass and flavor Fock spaces. Clearly, such inequivalence disappears for $\theta=0 \mathrm{and} /$ or $m_{2}=m_{1}$, as expected in the absence of field mixing.

To figure out the effects of the dynamical map (A7) on the flavor vacuum, let us focus on the second quantization of the flavor fields $\Psi_{e, \mu}$. As shown in Sect. 3, in the planewave representation the transformations (A1) yield the free-field like expansion (40), here rewritten for matter of convenience

$$
\begin{gathered}
\Psi_{\ell}(t, \mathbf{x})=\sum_{r=1,2} \int d^{3} k N\left[a_{\mathbf{k}, \ell}^{r}(\theta, t) u_{\mathbf{k}, \sigma}^{r}(t) e^{i \mathbf{k} \cdot \mathbf{x}}\right. \\
\left.+b_{\mathbf{k}, \ell}^{r \dagger}(\theta, t) v_{\mathbf{k}, \sigma}^{r}(t) e^{-i \mathbf{k} \cdot \mathbf{x}}\right] \\
(\ell, \sigma)=\{(e, 1),(\mu, 2)\},
\end{gathered}
$$

where the notation has been set in Sect. 3. The timedependent flavor annihilators are defined by [32]

$$
\begin{aligned}
a_{\mathbf{k}, \ell}^{r}(\theta, t) & =G_{\theta}^{-1}(t) a_{\mathbf{k}, \sigma}^{r} G_{\theta}(t), \quad(\ell, \sigma) \\
& =\{(e, 1),(\mu, 2)\},
\end{aligned}
$$

and similarly for $b_{\mathbf{k}, \ell}^{r}(\theta, t)$. To streamline the notation, henceforth we shall denote these operators by $a_{\mathbf{k}, \ell}^{r}(\theta, t) \equiv a_{\mathbf{k}, \ell}^{r}$. By computing the above product explicitly, we get

$$
\begin{aligned}
a_{\mathbf{k}, e}^{r}= & \cos \theta a_{\mathbf{k}, 1}^{r}+\sin \theta \sum_{s=1,2}\left[\left(u_{\mathbf{k}, 1}^{r \dagger}(t), u_{\mathbf{k}, 2}^{s}(t)\right) a_{\mathbf{k}, 2}^{s}\right. \\
& \left.+\left(u_{\mathbf{k}, 1}^{r \dagger}(t), v_{-\mathbf{k}, 2}^{s}(t)\right) b_{-\mathbf{k}, 2}^{s \dagger}\right], \\
b_{\mathbf{k}, e}^{r}= & \cos \theta b_{\mathbf{k}, 1}^{r}+\sin \theta \sum_{s=1,2}\left[\left(v_{\mathbf{k}, 2}^{s \dagger}(t), v_{\mathbf{k}, 1}^{r}(t)\right) b_{\mathbf{k}, 2}^{s}\right. \\
& \left.+\left(u_{-\mathbf{k}, 2}^{s \dagger}(t), v_{\mathbf{k}, 1}^{r}(t)\right) a_{-\mathbf{k}, 2}^{s \dagger}\right],
\end{aligned}
$$

(and similarly for $a_{\mathbf{k}, \mu}^{r}$ and $b_{\mathbf{k}, \mu}^{r}$ ). Therefore, we find that the flavor annihilators are related to the ladders operators in the mass representations via the combinations of two transformations: the standard Pontecorvo rotation (parameterized by the mixing angle $\theta$ ) and a Bogoliubov transformation (the terms in the square brackets) of coefficients ${ }^{4}$

$$
\begin{aligned}
V_{\mathbf{k}}^{11}(t) & \equiv\left(u_{\mathbf{k}, 1}^{1 \dagger}(t), v_{-\mathbf{k}, 2}^{1}(t)\right) \\
& =-e^{i\left(\omega_{\mathbf{k}, 1}+\omega_{\mathbf{k}, 2}\right) t} \frac{k_{z}\left(m_{1}+\omega_{\mathbf{k}, 1}-m_{2}-\omega_{\mathbf{k}, 2}\right)}{2 \sqrt{\omega_{\mathbf{k}, 1} \omega_{\mathbf{k}, 2}\left(m_{1}+\omega_{\mathbf{k}, 1}\right)\left(m_{2}+\omega_{\mathbf{k}, 2}\right)}},
\end{aligned}
$$

$V_{\mathbf{k}}^{22}(t) \equiv\left(u_{\mathbf{k}, 1}^{2 \dagger}(t), v_{-\mathbf{k}, 2}^{2}(t)\right)=-V_{\mathbf{k}}^{11}(t)$,

$V_{\mathbf{k}}^{12}(t) \equiv\left(u_{\mathbf{k}, 1}^{1 \dagger}(t), v_{-\mathbf{k}, 2}^{2}(t)\right)$

$$
=-e^{i\left(\omega_{\mathbf{k}, 1}+\omega_{\mathbf{k}, 2}\right) t} \frac{\left(k_{x}-i k_{y}\right)\left(m_{1}+\omega_{\mathbf{k}, 1}-m_{2}-\omega_{\mathbf{k}, 2}\right)}{2 \sqrt{\omega_{\mathbf{k} 1} \omega_{\mathbf{k}, 2}\left(m_{1}+\omega_{\mathbf{k}, 1}\right)\left(m_{2}+\omega_{\mathbf{k}, 2}\right)}},
$$

$$
\begin{aligned}
V_{\mathbf{k}}^{21}(t) & \equiv\left(u_{\mathbf{k}, 1}^{2 \dagger}(t), v_{-\mathbf{k}, 2}^{1}(t)\right) \\
& =-e^{i\left(\omega_{\mathbf{k}, 1}+\omega_{\mathbf{k}, 2}\right) t} \frac{\left(k_{x}+i k_{y}\right)\left(m_{1}+\omega_{\mathbf{k}, 1}-m_{2}-\omega_{\mathbf{k}, 2}\right)}{2 \sqrt{\omega_{\mathbf{k}, 1} \omega_{\mathbf{k}, 2}\left(m_{1}+\omega_{\mathbf{k}, 1}\right)\left(m_{2}+\omega_{\mathbf{k}, 2}\right)}}, \\
U_{\mathbf{k}}(t) & \equiv\left(v_{-\mathbf{k}, 2}^{s \dagger}(t), v_{-\mathbf{k}, 1}^{r}(t)\right)=\left(v_{\mathbf{k}, 2}^{s \dagger}(t), v_{\mathbf{k}, 1}^{r}(t)\right) \\
& =e^{i\left(\omega_{\mathbf{k}, 1}-\omega_{\mathbf{k}, 2}\right) t} \frac{\left[|\mathbf{k}|^{2}+\left(m_{1}+\omega_{\mathbf{k}, 1}\right)\left(m_{2}+\omega_{\mathbf{k}, 2}\right)\right]}{2 \sqrt{\omega_{\mathbf{k}, 1} \omega_{\mathbf{k}, 2}\left(m_{1}+\omega_{\mathbf{k}, 1}\right)\left(m_{2}+\omega_{\mathbf{k}, 2}\right)}} \delta_{r s s} .
\end{aligned}
$$

We notice that the above coefficients are non-vanishing, since (anti-)particle spinors with different masses are not orthogonal to each other. From the above relation, we also derive

$V_{\mathbf{k}}^{r 1}(t) V_{\mathbf{k}}^{s 1 *}(t)+V_{\mathbf{k}}^{r 2}(t) V_{\mathbf{k}}^{s 2 *}(t)=\left|V_{\mathbf{k}}\right|^{2} \delta_{r s}$,

where

$$
\begin{aligned}
\left|V_{\mathbf{k}}\right|^{2}= & {\left[( \frac { \omega _ { \mathbf { k } , 1 } + m _ { 1 } } { 2 \omega _ { \mathbf { k } , 1 } } ) ^ { \frac { 1 } { 2 } } ( \frac { \omega _ { \mathbf { k } , 2 } + m _ { 2 } } { 2 \omega _ { \mathbf { k } , 2 } } ) ^ { \frac { 1 } { 2 } } \left(\frac{|\mathbf{k}|}{\omega_{\mathbf{k}, 2}+m_{2}}\right.\right.} \\
& \left.\left.-\frac{|\mathbf{k}|}{\omega_{\mathbf{k}, 1}+m_{1}}\right)\right]^{2} .
\end{aligned}
$$

It is straightforward to show that

$\left|U_{\mathbf{k}}\right|^{2}+\left|V_{\mathbf{k}}\right|^{2}=1$,

\footnotetext{
${ }^{4}$ Here we only display the coefficients that explicitly appear in the computations of Sect. 3 .
} 
which guarantees that the flavor operators (A11) and (A12) and their hermitian conjugates are still canonical (at equal times) [32].

The appearance of a nontrivial Bogoliubov transformation in Eqs. (A11) and (A12) makes it clear that the flavor annihilators $a_{\mathbf{k}, e}^{r}$ and $b_{\mathbf{k}, e}^{r}$ do not actually annihilate the mass vacuum. In other terms, the vacua for flavor and mass fields (and the related Fock spaces) are not equivalent to each other, the former becoming a condensate of entangled massive particle/antiparticle pairs with density

$$
\begin{aligned}
& e, \mu \\
& \left\langle 0(\theta, t)\left|a_{\mathbf{k}, i}^{r \dagger} a_{\mathbf{k}, i}^{r}\right| 0(\theta, t)\right\rangle_{e, \mu} \\
& =\sin ^{2} \theta\left|V_{\mathbf{k}}\right|^{2}, \quad \forall t, i=1,2 .
\end{aligned}
$$

This is even more evident if we look at the explicit expression of $|0(\theta, t)\rangle_{e, \mu}$ in terms of $|0\rangle_{1,2}$. For instance, in the reference frame where $\mathbf{k}=(0,0, k)$, this reads

$$
\begin{aligned}
& |0(\theta, t)\rangle_{e, \mu} \\
& =\prod_{k} \prod_{r}\left[\left(1-\sin ^{2} \theta\left|V_{\mathbf{k}}\right|^{2}\right)\right. \\
& \quad-\varepsilon^{r} \sin \theta \cos \theta V_{\mathbf{k}}\left(a_{\mathbf{k}, 1}^{r \dagger} b_{-\mathbf{k}, 2}^{r \dagger}+a_{\mathbf{k}, 2}^{r \dagger} \beta_{-\mathbf{k}, 1}^{r \dagger}\right) \\
& \quad+\varepsilon^{r} \sin ^{2} \theta V_{\mathbf{k}}\left(U_{\mathbf{k}}^{*} \alpha_{\mathbf{k}, 1}^{r \dagger} b_{-\mathbf{k}, 1}^{r \dagger}-U_{\mathbf{k}} a_{\mathbf{k}, 2}^{r \dagger} b_{-\mathbf{k}, 2}^{r \dagger}\right) \\
& \left.\quad+\sin ^{2} \theta V_{\mathbf{k}}^{2} a_{\mathbf{k}, 1}^{r \dagger} b_{-\mathbf{k}, 2}^{r \dagger} a_{\mathbf{k}, 2}^{r \dagger} b_{-\mathbf{k}, 1}^{r \dagger}\right]|0\rangle_{1,2},
\end{aligned}
$$

where $\varepsilon^{r}=(-1)^{r}$ and we have omitted for simplicity the time-dependence in the r.h.s. This relation shows that the flavor vacuum is made up of pairwise correlated particles/antiparticles of both equal and different masses and opposite momenta. It is worth noting that the presence of different types of pairs makes this state different from the ground state of the BCS theory of superconductivity [85], where only one type of pair is involved.

Again, the density (A21) vanishes for $\theta=0$ and/or $m_{2}=m_{1}$, thus giving back the expected equality between the flavor and mass representations when there is no mixing. The same occurs in the limit of large momenta with respect to the masses $m_{1}$ and $m_{2}$, consistently with the recovery of the standard quantum mechanical picture.

Of course, by exploiting the symmetric structure of Eq. (A10), one can reverse the above reasoning and study the inherent structure of the mass vacuum in terms of the definite flavor quanta. What is obtained in that case is that such state would appear as a condensate of particle/antiparticle pairs of both equal and different flavors. For more details on the QFT formalism of field mixing and the related physical implications, we refer the reader to $[32,40,41,84]$. Specifically, in [40] it has been shown that the flavor Fock space cannot be obtained by the direct product of the Fock spaces for massive fields. Therefore, the nontrivial nature of QFT mixing is a feature boiling down to the nonfactorizability of the flavor states in terms of those with definite masses, including the vacuum. The entanglement content of the flavor vacuum has been quantified in [41] for the case of boson mixing and in the limit of relatively small difference of masses.

\section{Appendix B: Modified Unruh distribution for mixed fields}

In this Appendix we give more details on the calculation of the integral (51)

$$
\begin{aligned}
\mathcal{N}_{j, \kappa, \sigma}^{F G} \equiv & \sum_{r=1,2} \int d k_{x} F_{j, r, \sigma}\left(k_{x}, \Omega\right)\left[G_{j, 1, \sigma}^{*}\left(-k_{x}, \Omega\right) V_{\mathbf{k}}^{r 1}\right. \\
& \left.+G_{j, 2, \sigma}^{*}\left(-k_{x}, \Omega\right) V_{\mathbf{k}}^{r 2}\right] U_{\mathbf{k}}
\end{aligned}
$$

for spin $j=1$ and flavor $\ell=e$ (clearly, similarly considerations hold for $j=2$ and/or $\ell=\mu$ ). By setting $\epsilon \equiv \sqrt{m_{2}^{2}-m_{1}^{2}} / \omega_{\mathbf{k}, 1}$ and expanding around $\epsilon \ll 1$, to the leading order one can prove that

$\mathcal{N}_{1, \kappa, 1}^{F G} \simeq \int d k_{x} \epsilon^{2} \mathcal{I}_{\kappa}\left(k_{x}\right)$,

where

$$
\begin{aligned}
\mathcal{I}_{\kappa}\left(k_{x}\right) & \frac{i e^{-\pi \Omega}}{64 m_{1} \mu_{\vec{k}, 1} \omega_{\mathbf{k}, 1}^{4}}\left(\frac{\omega_{\mathbf{k}, 1}+k_{x}}{\omega_{\mathbf{k}, 1}-k_{x}}\right)^{i \Omega}\left(1+\frac{|\mathbf{k}|^{2}}{\left(m_{1}+\omega_{\mathbf{k}, 1}\right)^{2}}\right) \\
& \times\left\{k _ { z } ^ { 2 } ( \mu _ { \vec { k } , 1 } + ( \omega _ { \mathbf { k } , 1 } + k _ { x } ) \sqrt { \frac { \omega _ { \mathbf { k } , 1 } + k _ { x } } { \omega _ { \mathbf { k } , 1 } - k _ { x } } } ) \left[i \left(k_{x}\right.\right.\right. \\
& \left.-i k_{y}\right)\left(\mu_{\vec{k}, 1}+\left(\omega_{\mathbf{k}, 1}+2 m_{1}-k_{x}\right) \sqrt{\frac{\omega_{\mathbf{k}, 1}-k_{x}}{\omega_{\mathbf{k}, 1}+k_{x}}}\right) \\
& +\left[\mu_{\vec{k}, 1}\left(k_{x}-i k_{y}+m_{1}+\omega_{\mathbf{k}, 1}\right)+\sqrt{\frac{\omega_{\mathbf{k}, 1}-k_{x}}{\omega_{\mathbf{k}, 1}+k_{x}}}\left(k_{z}^{2}\right.\right. \\
& \left.\left.\left.+\left(k_{y}-i m_{1}\right)\left(i k_{x}+k_{y}-i\left(m_{1}+\omega_{\mathbf{k}, 1}\right)\right)\right)\right]\right] \\
& +\left[\mu_{\vec{k}, 1}\left(k_{x}-i k_{y}-m_{1}-\omega_{\mathbf{k}, 1}\right)\right. \\
& -\sqrt{\frac{\omega_{\mathbf{k}, 1}+k_{x}}{\omega_{\mathbf{k}, 1}-k_{x}}}\left(k_{y}^{2}+k_{z}^{2}+k_{x}\left(i k_{y}+m_{1}\right)\right. \\
& \left.\left.+i k_{y} \omega_{\mathbf{k}, 1}+m_{1}\left(m_{1}+\omega_{\mathbf{k}, 1}\right)\right)\right] \\
& \times\left[-i k_{z}^{2}\left(\mu_{\vec{k}, 1}+\left(\omega_{\mathbf{k}, 1}+2 m_{1}-k_{x}\right) \sqrt{\frac{\omega_{\mathbf{k}, 1}-k_{x}}{\omega_{\mathbf{k}, 1}+k_{x}}}\right)\right. \\
& +\left(k_{x}+i k_{y}\right)\left(\mu_{\vec{k}, 1}\left(k_{x}-i k_{y}+m_{1}+\omega_{\mathbf{k}, 1}\right)\right.
\end{aligned}
$$




$$
\begin{aligned}
& +\sqrt{\frac{\omega_{\mathbf{k}, 1}-k_{x}}{\omega_{\mathbf{k}, 1}+k_{x}}}\left(k_{z}^{2}\right. \\
& \left.\left.\left.\left.+\left(k_{y}-i m_{1}\right)\left(i k_{x}+k_{y}-i\left(m_{1}+\omega_{\mathbf{k}, 1}\right)\right)\right)\right)\right]\right\} .
\end{aligned}
$$

Due to the nontrivial dependence of $\mathcal{I}_{\kappa}$ by $k_{x}$, the integral (B2) cannot be solved analytically. However, for our purposes it is enough to note that $\mathcal{N}_{1, \kappa, 1}^{F G}$ can be rewritten equivalently as

$\mathcal{N}_{1, \kappa, 1}^{F G} \simeq \frac{\left|\Delta m^{2}\right|}{\mu_{\vec{k}, 1}^{2}} e^{-\pi \Omega} \mathcal{H}\left(\mu_{\vec{k}, 1}\right)$,

where we have separated out the dependence on both the mass difference $\left|\Delta m^{2}\right|$ and the Rindler energy $\Omega$. $\mathcal{H}\left(\mu_{\vec{k}, 1}\right)$ is a shorthand notation for the (dimensionless) $k_{x}$-integral of the residual function. ${ }^{5}$

The integral (B2) can be estimated numerically. In the limit of relativistic neutrinos and for values of $\Omega$ such that the unmodified Fermi-Dirac spectrum in Eq. (48) differs appreciably from zero, $\mathcal{N}_{j, \kappa, \sigma}^{F G}$ is finite and its real part assumes positive values. As $\Omega$ increases, the rapidly oscillating behavior of $\mathcal{H}\left(\mu_{\vec{k}, 1}\right)$ makes it difficult even numerical evaluations. However, this regime turns out to be less relevant, since the larger the energy $\Omega$, the lower the expected number of particles in the vacuum condensate. Further investigation of these technicalities is under active consideration and will be carried out elsewhere.

\section{References}

1. W.G. Unruh, Phys. Rev. D 14, 870 (1976)

2. P.M. Alsing, G.J. Milburn, Phys. Rev. Lett. 91, 180404 (2003)

3. I. Fuentes-Schuller, R.B. Mann, Phys. Rev. Lett. 95, 120404 (2005)

4. P.M. Alsing, I. Fuentes-Schuller, R.B. Mann, T.E. Tessier, Phys. Rev. A 74, 032326 (2006)

5. G. Adesso, I. Fuentes-Schuller, M. Ericsson, Phys. Rev. A 76, $062112(2007)$

6. P.C. Davies, Chaos 11, 539547 (2001)

7. S.R. Das, A. Zelnikov, Phys. Rev. D 64, 104001 (2001)

8. J. Giné, G.G. Luciano, Eur. Phys. J. C 80, 1039 (2020)

9. D.A.T. Vanzella, G.E.A. Matsas, Phys. Rev. Lett. 87, 151301 (2001)

10. A. Noto, R. Passante, Phys. Rev. D 88, 025041 (2013)

11. D.V. Ahluwalia, L. Labun, G. Torrieri, Eur. Phys. J. A 52, 189 (2016)

12. F. Scardigli, M. Blasone, G. Luciano, R. Casadio, Eur. Phys. J. C 78, $728(2018)$

13. F. Becattini, Phys. Rev. D 97, 085013 (2018)

14. G.G. Luciano, L. Petruzziello, Eur. Phys. J. C 79, 283 (2019)

15. M. Blasone, G. Lambiase, G.G. Luciano, L. Petruzziello, Phys. Rev. D 97, 105008 (2018)

${ }^{5}$ Strictly speaking, $\mathcal{H}\left(\mu_{\vec{k}, 1}\right)$ also depends on $\Omega$. Nevertheless, since the dependence is through an oscillating term, it does not affect significantly the outcome of the $k_{x}$-integral and can be in principle neglected.
16. G. Cozzella, S.A. Fulling, A.G.S. Landulfo, G.E.A. Matsas, D.A.T Vanzella, Phys. Rev. D 97, 105022 (2018)

17. M. Blasone, G. Lambiase, G.G. Luciano, L. Petruzziello, Phys. Lett. B 800, 135083 (2020)

18. M. Blasone, G. Lambiase, G.G. Luciano, L. Petruzziello, Eur. Phys. J. C 80, 130 (2020)

19. B.L. Torres, T.R. Perche, A.G.S. Landulfo, G.E.A. Matsas, Phys. Rev. D 102, 093003 (2020)

20. F. Hammad, A. Landry, D. Dijamco, Phys. Rev. D 103, 085010 (2021)

21. L.C.B. Crispino, A. Higuchi, G.E.A. Matsas, Rev. Mod. Phys. 80, 787 (2008)

22. R. Schutzhold, G. Schaller, D. Habs, Phys. Rev. Lett. 97, 121302 (2006)

23. K.K. Ng, L. Hodgkinson, J. Louko, R.B. Mann, E. MartinMartinez, Phys. Rev. D 90, 064003 (2014)

24. W.G. Unruh, Phys. Rev. Lett. 46, 1351 (1981)

25. S. Weinfurtner, E.W. Tedford, M.C.J. Penrice, W.G. Unruh, G.A. Lawrence, Phys. Rev. Lett. 106, 021302 (2011)

26. A. Retzker, J.I. Cirac, M.B. Plenio, B. Reznik, Phys. Rev. Lett. 101, $110402(2008)$

27. A. Iorio, G. Lambiase, Phys. Lett. B 716, 334 (2012)

28. C. Barcelo, S. Liberati, M. Visser, Living Rev. Relativ. 8, 12 (2005)

29. J. Marino, A. Noto, R. Passante, Phys. Rev. Lett. 113, 020403 (2014)

30. M. Blasone, G. Lambiase, G.G. Luciano, Phys. Rev. D 96, 025023 (2017)

31. M. Blasone, G. Lambiase, G.G. Luciano, J. Phys. Conf. Ser. 956, 012021 (2018)

32. M. Blasone, G. Vitiello, Ann. Phys. 244, 283 (1995)

33. M. Blasone, A. Capolupo, O. Romei, G. Vitiello, Phys. Rev. D 63, 125015 (2001)

34. G.G. Luciano, M. Blasone, Phys. Rev. D 104, 045004 (2021)

35. C. Tsallis, J. Stat. Phys. 52, 479 (1988)

36. C. Tsallis, Introduction to Non-Extensive Statistical Mechanics: Approaching a Complex World (Springer, Berlin, 2009)

37. C. Tsallis, L.J.L. Cirto, Eur. Phys. J. C 73, 2487 (2013)

38. M.L. Lyra, C. Tsallis, Phys. Rev. Lett. 80, 53 (1998)

39. J. Havrda, F. Charvát, Kybernetika 3, 1 (1967)

40. A. Cabo Montes de Oca, N.G. Cabo Bizet, arXiv:2005.07758 [hep$\mathrm{ph}]$

41. M. Blasone, F. Illuminati, G.G. Luciano, L. Petruzziello, Phys. Rev. A 103, 032434 (2021)

42. A.R. Plastino, A. Plastino, Phys. Lett. A 174, 384 (1993)

43. V.H. Hamity, D.E. Barraco, Phys. Rev. Lett. 76, 4664 (1996)

44. C. Tsallis, F.C. Sa Barreto, E.D. Loh, Phys. Rev. E 52, 1447 (1995)

45. A.R. Plastino, A. Plastino, H. Vucetich, Phys. Lett. A 207, 42 (1995)

46. G. Kaniadakis, A. Lavagno, P. Quarati, Phys. Lett. B 369, 308 (1996)

47. P. Jizba, J. Korbel, V. Zatloukal, Phys. Rev. E 95, 022103 (2017)

48. S. Nojiri, S.D. Odintsov, E.N. Saridakis, Eur. Phys. J. C 79, 242 (2019)

49. E.M. Barboza, Jr., R. Nunes, E.M.C. Abreu, J. Ananias Neto, Phys. A 436, 301 (2015)

50. V.F. Mukhanov, S. Winitzki, Introduction to Quantum Effects in Gravity (Cambridge University Press, Cambridge, 2007)

51. W. Greiner, J. Reinhardt, Field Quantization (Springer, Berlin, 1996)

52. D. Oriti, Nuovo Cim. B 115, 1005 (2000)

53. U.H. Gerlach, Phys. Rev. D 38, 514 (1988)

54. S. Takagi, Prog. Theor. Phys. Suppl. 88, 1 (1986)

55. S.M. Bilenky, B. Pontecorvo, Phys. Rep. 41, 225 (1978)

56. C. Giunti, C.W. Kim, Fundamentals of Neutrino Physics and Astrophysics (Oxford Univ. Press, Oxford, 2007) 
57. Y. Fukuda et al. [Super-Kamiokande], Phys. Rev. Lett. 81, 1562 (1998)

58. K. Abe et al. [Super-Kamiokande], Phys. Rev. Lett. 110, 181802 (2013)

59. Q.R. Ahmad et al. [SNO], Phys. Rev. Lett. 87, 071301 (2001)

60. Q.R. Ahmad et al. [SNO], Phys. Rev. Lett. 89, 011301 (2002)

61. M. Blasone, G. Lambiase, G.G. Luciano, J. Phys. Conf. Ser. 631, 012053 (2015)

62. M. Blasone, G. Lambiase, G.G. Luciano, J. Phys. Conf. Ser. 880, 012043 (2017)

63. S. Abe, S. Martínez, F. Pennini, A. Plastino, Phys. Lett. A 281, 126 (2001)

64. F. Büyükkiliç, D. Demirhan, Phys. Lett. A 181, 24 (1993)

65. F. Büyükkiliç, D. Demirhan, A. Güleç, Phys. Lett. A 197, 209 (1995)

66. U. Timakli, F. Büyükkiliç, D. Demirhan, Phys. A 240, 657 (1997)

67. Q.A. Wang, M. Pezeril, A. Le Mèhautè, Phys. A 278, 337 (2000)

68. J. Chen, Z. Zhang, G. Su, L. Chen, Y. Shu, Phys. Lett. A 300, 65 (2002)

69. F. Pennini, A. Plastino, A.R. Plastino, Phys. A 234, 471 (1996)

70. Q.A. Wang, L. Mèhautè, Phys. Lett. A 235, 222 (1997)

71. W.M. Alberico, A. Lavagno, P. Quarati, Eur. Phys. J. C 12, 499 (2000)
72. H. Shababi, K. Ourabah, Eur. Phys. J. Plus 135, 697 (2020)

73. G.G. Luciano, Eur. Phys. J. C 81, 672 (2021)

74. A. Lavagno, P. Narayana Swamy, Phys. Rev. E 65, 036101 (2002)

75. F. Jackson, Mess. Math. 38, 57 (1909)

76. B. Mirza, H. Mohammadzadeh, J. Phys. A Math. Theor. 44, 475003 (2011)

77. S. Abe, A.K. Rajagopal, Phys. Rev. A 60, 3461 (1999)

78. C. Tsallis, S. Lloyd, M. Baranger, Phys. Rev. A 63, 042104 (2001)

79. N. Canosa, R. Rossignoli, Phys. Rev. Lett. 88, 170401 (2002)

80. G. Kaniadakis, Phys. Rev. E 66, 056125 (2002)

81. N. Drepanou, A. Lymperis, E.N. Saridakis, K. Yesmakhanova, arXiv:2109.09181 [gr-qc]

82. A.C. Sparavigna, Int. J. Sci. 4, 47 (2015)

83. P. Longhi, R. Soldati, Int. J. Mod. Phys. A 28, 1350109 (2013)

84. M. Blasone, A. Capolupo, G. Vitiello, Phys. Rev. D 66, 025033 (2002)

85. J. Bardeen, L.N. Cooper, J.R. Schrieffer, Phys. Rev. 106, 162 (1957) 